\title{
Dark matter and flavor changing in the flipped 3-3-1 model
}

\author{
D.T. Huong, ${ }^{a}$ D.N. Dinh, ${ }^{a}$ L.D. Thien ${ }^{b, c}$ and Phung Van Dong ${ }^{c, 1}$ \\ ${ }^{a}$ Institute of Physics, Vietnam Academy of Science and Technology, \\ 10 Dao Tan, Ba Dinh, Hanoi, Vietnam \\ ${ }^{b}$ Graduate University of Science and Technology, Vietnam Academy of Science and Technology, \\ 18 Hoang Quoc Viet, Cau Giay, Hanoi, Vietnam \\ ${ }^{c}$ Phenikaa Institute for Advanced Study and Faculty of Basic Science, Phenikaa University, \\ To Huu, Yen Nghia, Ha Dong, Hanoi 100000, Vietnam \\ E-mail: dthuong@iop.vast.ac.vn, dndinh@iop.vast.ac.vn, \\ ldthien@grad.iop.vast.ac.vn, dong.phungvan@phenikaa-uni.edu.vn
}

ABSTRACT: The flipped 3-3-1 model discriminates lepton families instead of the quark ones in normal sense, where the left-handed leptons are in two triplets plus one sextet while the left-handed quarks are in antitriplets, under $\mathrm{SU}(3)_{L}$. We investigate a minimal setup of this model and determine novel consequences of dark matter stability, neutrino mass generation, and lepton flavor violation. Indeed, the model conserves a noncommutative $B-L$ symmetry, which prevents the unwanted vacua and interactions and provides the matter parity and dark matter candidates that along with normal matter form gauge multiplets. The neutrinos obtain suitable masses via a type I and II seesaw mechanism. The nonuniversal couplings of $Z^{\prime}$ with leptons govern lepton flavor violating processes such as $\mu \rightarrow 3 e, \mu \rightarrow e \bar{\nu}_{\mu} \nu_{e}, \mu-e$ conversion in nuclei, semileptonic $\tau \rightarrow \mu(e)$ decays, as well as the nonstandard interactions of neutrinos with matter. This $Z^{\prime}$ may also set the dark matter observables and give rise to the LHC dilepton and dijet signals.

Keywords: Neutrino Physics, Cosmology of Theories beyond the SM, Gauge Symmetry

ARXIV EPRINT: 1906.05240

\footnotetext{
${ }^{1}$ Corresponding author.
} 


\section{Contents}

1 Introduction 1

2 Novel features of the model 2

2.1 Proposal 2

2.2 Dark matter 4

2.3 Lagrangian 5

2.4 Neutrino mass $\quad 7$

2.5 Gauge sector 8

$\begin{array}{lll}3 & \text { FCNC } & 9\end{array}$

4 Phenomenology 12

4.1 Leptonic three-body decays 12

4.1.1 $\tau^{+} \rightarrow \mu^{+} \mu^{+} \mu^{-}, \tau^{+} \rightarrow e^{+} e^{+} e^{-}$and $\mu^{+} \rightarrow e^{+} e^{+} e^{-} \quad 12$

$\begin{array}{lll}4.1 .2 & \tau^{+} \rightarrow \mu^{+} e^{+} e^{-} \text {and } \tau^{+} \rightarrow e^{+} \mu^{+} \mu^{-} & 14\end{array}$

$\begin{array}{lll}\text { 4.1.3 } & \tau^{+} \rightarrow \mu^{+} \mu^{+} e^{-} \text {and } \tau^{+} \rightarrow e^{+} e^{+} \mu^{-} & 15\end{array}$

$\begin{array}{lll}\text { 4.1.4 Comment on wrong } \mu \text { and } \tau \text { decays } & 17\end{array}$

4.2 Semileptonic $\tau \rightarrow \mu$ and $\tau \rightarrow e$ decays $\quad 17$

$4.3 \mu-e$ conversion in nuclei 20

4.4 Constraining nonstandard neutrino interactions 22

4.5 LHC dilepton and dijet searches 24

$\begin{array}{ll}4.6 & \text { Dark matter } \\ 25\end{array}$

5 Conclusion $\quad 26$

\section{Introduction}

The $\mathrm{SU}(3)_{C} \otimes \mathrm{SU}(3)_{L} \otimes \mathrm{U}(1)_{X}(3-3-1)$ gauge model has been extensively studied over the last decades [1-6]. Indeed, it can resolve the profound questions of fermion generation number due to $\mathrm{SU}(3)_{L}$ anomaly cancellation (see, for instance, $[2,4]$ ), electric charge quantization due to $\mathrm{SU}(3)_{L}$ particle structure (see, for instance, [7-11]), and strong CP conservation due to automatic Peccei-Quinn like symmetry (see, for instance, [12-15]). Additionally, the model can supply consistent neutrino masses [16-31] and viable dark matter candidates [3250]. The (Higgs) inflation scenarios and leptogenesis mechanism can be newly recognized in this setup, which produce the accelerated expansion of the early universe and the baryon asymmetry of the universe, respectively [51-53].

The 3-3-1 model has been well established by assigning one of quark generations to transform under $\mathrm{SU}(3)_{L}$ differently from other quark generations, whereas all lepton generations transform identically under this group. This is required in order to cancel the 
$\left[\mathrm{SU}(3)_{L}\right]^{3}$ anomaly [54-57]. Recently, Fonseca and Hirsch [58] have made an intriguing observation of a flipped 3-3-1 model, in which one of lepton generations is arranged differently from the remaining lepton generations, while all quark generations are identical under $\mathrm{SU}(3)_{L}$, by contrast. This flip of quark and lepton representations converts the flavor matters in quark sector [59-66] to the lepton sector. In this case, the neutral current of $Z^{\prime}$ conserves quark flavors, while it violates lepton flavors. Therefore, the tree-level lepton flavor violating processes, for instance $\mu \rightarrow 3 e$ and $\mu \rightarrow e \bar{\nu}_{\mu} \nu_{e}$ [58], exist due to the exchange of $Z^{\prime}$. This flavor changing also leads to the anomalies in interaction of neutrinos with matter.

As supposed in [58], the flipped 3-3-1 model realizes a special content of fermions. In this case, one can verify that the gravitational anomaly $[\text { Gravity }]^{2} \mathrm{U}(1)_{X}$ vanishes. This makes the model valid up to the Planck scale, where the effect of quantum gravity becomes important $[67,68]$. This condition is necessary in order to ensure a manifest $B-L$ symmetry at high energy, analogous to the standard model. Additionally, $B-L$ neither commutes nor closes algebraically with $\mathrm{SU}(3)_{L}$, similar to the electric charge. The algebraic closure condition results in a complete gauge symmetry $\mathrm{SU}(3)_{C} \otimes \mathrm{SU}(3)_{L} \otimes \mathrm{U}(1)_{X} \otimes \mathrm{U}(1)_{N}$, where $N$ determines $B-L$, in the same situation that $X$ defines the electric charge [44-48, 51, 52]. The $B-L$ breaking leads to a matter parity which characterizes and stabilizes dark matter candidates. It is noteworthy that dark matter is unified with normal matter in gauge multiplets due to the noncommutativity of $B-L$ symmetry. Additionally, the matter parity cures the unwanted vacua and interactions, which otherwise imply large flavor-changing neutral currents (FCNCs) in the lepton sector. The type I seesaw can be realized due to the $B-L$ dynamics. However, this model contains naturally type II and III seesaws too.

The rest of this work is organized as follows. In section 2 we examine the flipped 3-3-1 model when imposing the $B-L$ symmetry and matter parity. The dark matter candidates are identified. The masses of lepton sextet including neutrinos are obtained. The gauge sector is diagonalized. In section 3 we determine the tree-level FCNC coupled to $Z^{\prime}$. The setup also implies the tree-level FCNC coupled to the standard model Higgs boson, but it is subleading and neglected. In section 4, the lepton flavor violating processes, the nonstandard interactions of neutrinos with matter, the LHC dilepton and dijet searches, and the dark matter observables are obtained. Finally, we conclude this work in section 5 .

\section{Novel features of the model}

\subsection{Proposal}

As stated, the 3-3-1 gauge symmetry is given by

$$
\mathrm{SU}(3)_{C} \otimes \mathrm{SU}(3)_{L} \otimes \mathrm{U}(1)_{X}
$$

where the first factor is the usual QCD group, while the last two are a nontrivial extension of the electroweak group. The electric charge and hypercharge are embedded as

$$
Q=T_{3}+\beta T_{8}+X, \quad Y=\beta T_{8}+X
$$


where $T_{n}(n=1,2,3, \ldots, 8)$ and $X$ are $\mathrm{SU}(3)_{L}$ and $\mathrm{U}(1)_{X}$ generators, respectively. The coefficient $\beta$ determines the electric charge of new particles, which is arbitrary on the theoretical ground and independent of all the anomalies.

The new observation $[58]$ is that the $\left[\mathrm{SU}(3)_{L}\right]^{3}$ anomaly $(\mathcal{A})$ induced by a fermion sextet is related to that by a fermion triplet as $\mathcal{A}(6)=7 \mathcal{A}(3)$, where the color number is not counted. If one puts a lepton generation in a sextet and two other lepton generations in triplets, the anomaly contributed by the three lepton generations equals $9 \mathcal{A}(3)$. This cancels the contribution of three quark generations arranged in antitriplets, since $\mathcal{A}\left(3^{*}\right)=$ $-\mathcal{A}(3)$ and quarks have three colors. In general, it is proved that the generation number must be a multiple of three in order to embed left-handed fermion doublets in $\mathrm{SU}(3)_{L}$ representations while keeping right-handed fermions as singlets similar to the standard model, which provides a partial solution to the flavor question.

That said, the left-handed fermion representations under $\mathrm{SU}(3)_{L}$ are generally given by

$$
\begin{aligned}
& \psi_{1 L}=\left(\begin{array}{ccc}
\xi_{1}^{-q} & \frac{1}{\sqrt{2}} \xi_{2}^{-1-q} & \frac{1}{\sqrt{2}} \nu_{1} \\
\frac{1}{\sqrt{2}} \xi_{2}^{-1-q} & \xi_{3}^{-2-q} & \frac{1}{\sqrt{2}} e_{1} \\
\frac{1}{\sqrt{2}} \nu_{1} & \frac{1}{\sqrt{2}} e_{1} & k_{1}^{q}
\end{array}\right)_{L} \sim 6 \\
& \psi_{\alpha L}=\left(\begin{array}{c}
\nu_{\alpha} \\
e_{\alpha} \\
k_{\alpha}^{q}
\end{array}\right)_{L} \sim 3, \quad Q_{a L}=\left(\begin{array}{c}
d_{a} \\
-u_{a} \\
j_{a}^{-q-1 / 3}
\end{array}\right)_{L} \sim 3^{*},
\end{aligned}
$$

plus right-handed fermion singlets, $\xi_{a R}, e_{a R}, k_{a R}, u_{a R}, d_{a R}$, and $j_{a R}$. The generation indices are $\alpha=2,3$ and $a=1,2,3$. The new fields $\xi_{1,2,3}, k_{a}$, and $j_{a}$ possess electric charges as superscripted, which depend on a basic electric charge parameter $q=-(1+\sqrt{3} \beta) / 2$. It is easily checked that all the other anomalies vanish. An alternative version can be proposed, such that a lepton generation is in antisextet, two other lepton generations in antitriplets, and all the quark generations in triplets, under $\mathrm{SU}(3)_{L}$. However, this modification should be equivalently physical to the original proposal, which will be skipped.

The previous study [58] considered a special version, given that $q=-1$ or $\beta=1 / \sqrt{3}{ }^{1}$ In this case, $\xi_{2 R}^{0}$ is a 3-3-1 singlet which can be omitted as $\nu_{a R}$ are. Additionally, $\xi_{3 R}^{-}$can be discarded while $\xi_{3 L}^{-}$is replaced by $\left(\xi_{1 R}^{+}\right)^{c}=\xi_{1 L}^{-}$. This restriction results in the most

\footnotetext{
${ }^{1}$ The 3-3-1 model with $\beta=1 / \sqrt{3}$ has a Landau pole larger than the Planck scale, as expected [69, 70].
} 
economical model, explicitly written under the 3-3-1 symmetry as

$$
\begin{aligned}
\psi_{1 L} & =\left(\begin{array}{ccc}
\xi^{+} & \frac{1}{\sqrt{2}} \xi^{0} & \frac{1}{\sqrt{2}} \nu_{1} \\
\frac{1}{\sqrt{2}} \xi^{0} & \xi^{-} & \frac{1}{\sqrt{2}} e_{1} \\
\frac{1}{\sqrt{2}} \nu_{1} & \frac{1}{\sqrt{2}} e_{1} & E_{1}
\end{array}\right)_{L} \sim\left(1,6,-\frac{1}{3}\right), \\
\psi_{\alpha L} & =\left(\begin{array}{c}
\nu_{\alpha} \\
e_{\alpha} \\
E_{\alpha}
\end{array}\right)_{L} \sim\left(1,3,-\frac{2}{3}\right), \\
e_{a R} & \sim(1,1,-1), \quad E_{a R} \sim(1,1,-1), \\
Q_{a L} & =\left(\begin{array}{c}
d_{a} \\
-u_{a} \\
U_{a}
\end{array}\right)_{L} \sim\left(3,3^{*}, \frac{1}{3}\right), \\
u_{a R} & \sim(3,1,2 / 3), \quad d_{a R} \sim(3,1,-1 / 3), \quad U_{a R} \sim(3,1,2 / 3),
\end{aligned}
$$

where we relabel $k$ as $E$ and $j$ as $U$, since they have the same electric charge as of $e$ and $u$, respectively. The subscripts of $\xi$ are omitted without confusion. The authors of [58] pointed out that since the model cooperates a real triplet $\xi$ under $\mathrm{SU}(2)_{L}$, it can be made heavy in order to keep the model phenomenologically viable. Further, we will show that the simplest version provides dark matter candidates as well as neutrino masses naturally. Otherwise, for $q \neq-1$, the fermions $\xi$ must have right-handed components and gain light Dirac masses in the weak scale, which are strongly disfavored by the electroweak data.

The scalar content responsible for symmetry breaking and mass generation is given by

$$
\begin{aligned}
\eta=\left(\begin{array}{c}
\eta_{1}^{0} \\
\eta_{2}^{-} \\
\eta_{3}^{-}
\end{array}\right) \sim(1,3,-2 / 3), \quad \rho=\left(\begin{array}{c}
\rho_{1}^{+} \\
\rho_{2}^{0} \\
\rho_{3}^{0}
\end{array}\right) \sim(1,3,1 / 3), \\
\chi=\left(\begin{array}{c}
\chi_{1}^{+} \\
\chi_{2}^{0} \\
\chi_{3}^{0}
\end{array}\right) \sim(1,3,1 / 3), \quad S=\left(\begin{array}{ccc}
S_{11}^{++} & \frac{1}{\sqrt{2}} S_{12}^{+} & \frac{1}{\sqrt{2}} S_{13}^{+} \\
\frac{1}{\sqrt{2}} S_{12}^{+} & S_{22}^{0} & \frac{1}{\sqrt{2}} S_{23}^{0} \\
\frac{1}{\sqrt{2}} S_{13}^{+} & \frac{1}{\sqrt{2}} S_{23}^{0} & S_{33}^{0}
\end{array}\right) \sim(1,6,2 / 3) .
\end{aligned}
$$

Note that $\rho$ and $\chi$ are identical under the gauge symmetry, but distinct under the $B-L$ charge, as shown below.

\subsection{Dark matter}

First note that the electric charge neither commutes nor closes algebraically with $\mathrm{SU}(3)_{L}$, since $\left[Q, T_{n}\right] \neq 0$ for $n=1,2,4,5$ and $\operatorname{Tr} Q \neq 0$ for various particle multiplets. This property applies for every 3-3-1 model, including the standard model with $\mathrm{SU}(2)_{L}$.

Similarly to the electric charge, $B-L$ neither commutes nor closes algebraically with $\mathrm{SU}(3)_{L}$, which differs from the case of the standard model. Indeed, the standard model conserves $\mathrm{U}(1)_{B-L}$, which follows that $[B-L]\left(\xi^{+}\right)=[B-L]\left(\xi^{-}\right)=[B-L]\left(\xi^{0}\right) \equiv n$ for a 


\begin{tabular}{|lcccccccccccccc|}
\hline Multiplet & $\psi_{1 L}$ & $\psi_{\alpha L}$ & $Q_{a L}$ & $e_{a R}$ & $E_{a R}$ & $u_{a R}$ & $d_{a R}$ & $U_{a R}$ & $\eta$ & $\rho$ & $\chi$ & $S$ & $\nu_{a R}$ & $\phi$ \\
\hline$N$ & $-2 / 3$ & $-4 / 3$ & $2 / 3$ & -1 & -2 & $1 / 3$ & $1 / 3$ & $4 / 3$ & $-1 / 3$ & $-1 / 3$ & $2 / 3$ & $4 / 3$ & -1 & 2 \\
\hline
\end{tabular}

Table 1. $N$-charge of the model's multiplets.

\begin{tabular}{|lccccccccccccc|}
\hline Field & $\xi^{+}$ & $\xi^{0}$ & $\xi^{-}$ & $E^{-}$ & $U^{2 / 3}$ & $X^{+}$ & $Y^{0}$ & $\eta_{3}^{-}$ & $\rho_{3}^{0}$ & $\chi_{1}^{+}$ & $\chi_{2}^{0}$ & $S_{13}^{+}$ & $S_{23}^{0}$ \\
\hline$B-L$ & 0 & 0 & 0 & -2 & $4 / 3$ & 1 & 1 & -1 & -1 & 1 & 1 & 1 & 1 \\
$W_{P}$ & -1 & -1 & -1 & -1 & -1 & -1 & -1 & -1 & -1 & -1 & -1 & -1 & -1 \\
\hline
\end{tabular}

Table 2. Nontrivial matter parity and $B-L$ charge.

$\mathrm{SU}(2)_{L}$ triplet. Using the condition $[B-L]\left(\xi^{+}\right)=-[B-L]\left(\xi^{-}\right)$, we obtain $n=0$. Supposing that the 3-3-1 model conserves $B-L$ charge, we find $B-L=\operatorname{diag}(0,0,0,-1,-1,-2)$ for the sextet $\left(\xi^{+} \xi^{0} \xi^{-} \nu_{1} e_{1} E_{1}\right)_{L}$ and $B-L=\operatorname{diag}(-1,-1,-2)$ for the triplets $\left(\nu_{\alpha} e_{\alpha} E_{\alpha}\right)_{L}$. This implies that $\left[B-L, T_{n}\right] \neq 0$ for $n=4,5,6,7$ and $\operatorname{Tr}[B-L] \neq 0$, as expected.

The requirement of algebraic closure between $B-L$ and $\mathrm{SU}(3)_{L}$ results in an extra $\mathrm{U}(1)_{N}$ symmetry, such that

$$
B-L=\frac{2}{\sqrt{3}} T_{8}+N
$$

where $N$ determines $B-L$ in the same situation that $X$ does so for $Q[44-48,51,52]$. This way leads to the group structure $\mathrm{SU}(3)_{C} \otimes \mathrm{SU}(3)_{L} \otimes \mathrm{U}(1)_{X} \otimes \mathrm{U}(1)_{N}$, called 3-3-1-1. Additionally, $N$ and $B-L$ are gauged charges since they are related to the gauged charge $T_{8}$. The 3-3-1-1 gauge theory requires $\nu_{a R}$ in addition to the existing fermions in order to cancel the $B-L$ anomalies as well as a scalar field $\phi$ that couples to $\nu_{R} \nu_{R}$ and necessarily breaks $\mathrm{U}(1)_{N}$. The $N$-charges of multiplets are summarized in table 1

Assuming that $\mathrm{U}(1)_{N}$ is broken at high energy due to $\phi$, the heavy particles such as the $\mathrm{U}(1)_{N}$ gauge $(C)$ and Higgs $(\phi)$ bosons as well as right-handed neutrinos $\left(\nu_{a R}\right)$ are all integrated out. The imprint at low energy is only the 3-3-1 model, conserving the matter parity as residual gauge symmetry,

$$
W_{P}=(-1)^{3(B-L)+2 s}=(-1)^{2 \sqrt{3}\left(T_{8}+N\right)+2 s},
$$

which is defined by the vacuum of the mentioned $\mathrm{U}(1)_{N}$ breaking field [46].

Above, " $W$ " means the fields that have "wrong" $B-L$ charge in comparison to the standard model which transform nontrivially under the matter parity. They are collected in table 2. The remaining fields have $W_{P}=1$, called normal fields.

Because the matter parity is conserved, the lightest $W$-particle (LWP) is stabilized, responsible for dark matter. The dark matter candidates include a fermion $\xi^{0}$, a vector $Y^{0}$, and a combination of $\rho_{3}^{0}, \chi_{2}^{0}$, and $S_{23}^{0}$. Due to the gauge interaction, $Y^{0}$ annihilates completely into the standard model particles. The realistic candidates that have correct abundance are only the fermion or scalar, as shown below.

\subsection{Lagrangian}

Hereafter, we consider the theory at low energy that includes only the light fields. The contribution of heavy fields $\left(C, \phi, \nu_{a R}\right)$ is separately mentioned if necessary. 
The total Lagrangian consists of

$$
\mathcal{L}=\mathcal{L}_{\text {kinetic }}+\mathcal{L}_{\text {Yukawa }}-V
$$

where the first part composes the kinetic terms plus gauge interactions.

The second part includes Yukawa interactions, obtained by

$$
\begin{aligned}
\mathcal{L}_{\text {Yukawa }}= & h_{\alpha a}^{e} \bar{\psi}_{\alpha L} \rho e_{a R}+h_{\alpha a}^{E} \bar{\psi}_{\alpha L} \chi E_{a R}+h_{1 a}^{E} \bar{\psi}_{1 L} S E_{a R}+h^{\xi} \bar{\psi}_{1 L}^{c} \psi_{1 L} S \\
& +h_{a b}^{u} \bar{Q}_{a L} \rho^{*} u_{b R}+h_{a b}^{d} \bar{Q}_{a L} \eta^{*} d_{b R}+h_{a b}^{U} \bar{Q}_{a L} \chi^{*} U_{b R}+H . c .
\end{aligned}
$$

Note that the unwanted Yukawa interactions are

$$
\begin{aligned}
\text { fYukawa }= & s_{\alpha a}^{e} \bar{\psi}_{\alpha L} \chi e_{a R}+s_{1 a}^{e} \bar{\psi}_{1 L} S e_{a R}+s_{\alpha a}^{E} \bar{\psi}_{\alpha L} \rho E_{a R} \\
& +s_{a b}^{u} \bar{Q}_{a L} \chi^{*} u_{b R}+s_{a b}^{U} \bar{Q}_{a L} \rho^{*} U_{b R}+H . c .,
\end{aligned}
$$

which are suppressed due to the $\mathrm{U}(1)_{N}$ symmetry or matter parity at low energy. In the ordinary 3-3-1 models, they are present, characterizing an approximate $B-L$ symmetry. In other words, they should be small in comparison to the normal couplings $s \ll h$, respectively.

The last part is the scalar potential,

$$
\begin{aligned}
V= & \mu_{\eta}^{2} \eta^{\dagger} \eta+\mu_{\rho}^{2} \rho^{\dagger} \rho+\mu_{\chi}^{2} \chi^{\dagger} \chi+\mu_{S}^{2} \operatorname{Tr}\left(S^{\dagger} S\right) \\
& +\lambda_{\eta}\left(\eta^{\dagger} \eta\right)^{2}+\lambda_{\rho}\left(\rho^{\dagger} \rho\right)^{2}+\lambda_{\chi}\left(\chi^{\dagger} \chi\right)^{2}+\lambda_{1 S} \operatorname{Tr}^{2}\left(S^{\dagger} S\right)+\lambda_{2 S} \operatorname{Tr}\left(S^{\dagger} S\right)^{2} \\
& +\lambda_{\eta \rho}\left(\eta^{\dagger} \eta\right)\left(\rho^{\dagger} \rho\right)+\lambda_{\chi \eta}\left(\chi^{\dagger} \chi\right)\left(\eta^{\dagger} \eta\right)+\lambda_{\chi \rho}\left(\chi^{\dagger} \chi\right)\left(\rho^{\dagger} \rho\right) \\
& +\lambda_{\eta S}\left(\eta^{\dagger} \eta\right) \operatorname{Tr}\left(S^{\dagger} S\right)+\lambda_{\rho S}\left(\rho^{\dagger} \rho\right) \operatorname{Tr}\left(S^{\dagger} S\right)+\lambda_{\chi S}\left(\chi^{\dagger} \chi\right) \operatorname{Tr}\left(S^{\dagger} S\right) \\
& +\lambda_{\eta \rho}^{\prime}\left(\eta^{\dagger} \rho\right)\left(\rho^{\dagger} \eta\right)+\lambda_{\chi \eta}^{\prime}\left(\chi^{\dagger} \eta\right)\left(\eta^{\dagger} \chi\right)+\lambda_{\chi \rho}^{\prime}\left(\chi^{\dagger} \rho\right)\left(\rho^{\dagger} \chi\right) \\
& +\lambda_{\chi S}^{\prime}\left(\chi^{\dagger} S\right)\left(S^{\dagger} \chi\right)+\lambda_{\eta S}^{\prime}\left(\eta^{\dagger} S\right)\left(S^{\dagger} \eta\right)+\lambda_{\rho S}^{\prime}\left(\rho^{\dagger} S\right)\left(S^{\dagger} \rho\right) \\
& +\left(\mu \eta \rho \chi+\mu^{\prime} \chi^{T} S^{*} \chi+H . c .\right)
\end{aligned}
$$

Here the couplings $\lambda$ 's are dimensionless, while the parameters $\mu$ 's have mass dimension. The unwanted interactions include

$$
V=f^{2} \rho^{\dagger} \chi+f^{\prime} \rho^{T} S^{*} \chi+f^{\prime \prime} \rho^{T} S^{*} \rho+H . c .
$$

which are suppressed by the $\mathrm{U}(1)_{N}$ symmetry or matter parity at low energy, where $f$ 's are mass parameters analogous to $\mu$ 's. 


\subsection{Neutrino mass}

First note that due to the matter parity conservation, only the even scalars develop vacuum expectation values (VEVs), such that

$$
\begin{array}{ll}
\langle\eta\rangle=\frac{1}{\sqrt{2}}\left(\begin{array}{l}
u \\
0 \\
0
\end{array}\right), & \langle\rho\rangle=\frac{1}{\sqrt{2}}\left(\begin{array}{l}
0 \\
v \\
0
\end{array}\right), \\
\langle\chi\rangle=\frac{1}{\sqrt{2}}\left(\begin{array}{c}
0 \\
0 \\
w
\end{array}\right), & \langle S\rangle=\frac{1}{\sqrt{2}}\left(\begin{array}{ccc}
0 & 0 & 0 \\
0 & \kappa & 0 \\
0 & 0 & \Lambda
\end{array}\right) .
\end{array}
$$

Substituting the VEVs into the Yukawa Lagrangian, the quarks and exotic leptons gain suitable masses as follows

$$
\begin{aligned}
& {\left[m_{u}\right]_{a b}=\frac{h_{a b}^{u}}{\sqrt{2}} v,} \\
& {\left[m_{d}\right]_{a b}=-\frac{h_{a b}^{d}}{\sqrt{2}} u,} \\
& {\left[m_{U}\right]_{a b}=-\frac{h_{a b}^{U}}{\sqrt{2}} w,} \\
& m_{\xi}=-\sqrt{2} h^{\xi} \Lambda, \\
& {\left[m_{E}\right]_{1 b}=-\frac{h_{1 b}^{E}}{\sqrt{2}} \Lambda,} \\
& {\left[m_{E}\right]_{\alpha b}=-\frac{h_{\alpha b}^{E}}{\sqrt{2}} w .}
\end{aligned}
$$

The ordinary leptons obtain masses

$$
\left[m_{e}\right]_{\alpha b}=-\frac{h_{\alpha b}^{e}}{\sqrt{2}} v, \quad\left[m_{\nu}\right]_{11}=\sqrt{2} \kappa h^{\xi} .
$$

Note that the constraint from the $\rho$ parameter implies $\kappa \lesssim \mathcal{O}(1) \mathrm{GeV}$. Hence, a small mixing between $\xi^{ \pm}$and $E_{a}^{ \pm}$proportional to $\kappa$ can be neglected, since $\kappa \ll w, \Lambda$, where the last two VEVs are in the TeV scale. The fields $\mu$ and $\tau$ get desirable masses. However, the electron and last two neutrinos have vanishing masses, which are inconsistent.

The electron mass vanishes similarly to the original study, which can be radiatively induced [58]. An extra remark is that the interaction $\psi_{1 L} \psi_{1 L} S \supset\left(\xi^{+} \xi^{0} \xi^{-}\right)\left(\nu_{1 L} e_{1 L}\right)\left(S_{13}^{+} S_{23}^{0}\right)$ provides the need for a type III seesaw, where the mediator is a heavy fermion triplet, $\xi$. But the relevant neutrino $\left(\nu_{1}\right)$ mass generated is zero due to $\left\langle S_{23}^{0}\right\rangle=0$ resulting from the matter parity conservation, which does not change the above value of a type II seesaw.

As mentioned, the heavy fields $\phi, \nu_{R}$ are present and can imply neutrino masses via

$$
\mathcal{L}_{\nu}=h_{\alpha b}^{\nu} \bar{\psi}_{\alpha L} \eta \nu_{b R}+\frac{1}{2} h_{a b}^{R} \bar{\nu}_{a R}^{c} \nu_{b R} \phi+H . c .
$$

We achieve Dirac masses $\left[m_{\nu}^{D}\right]_{\alpha b}=-h_{\alpha b}^{\nu} u / \sqrt{2}$ and Majorana masses $\left[m_{\nu}^{R}\right]_{a b}=-h_{a b}^{R}\langle\phi\rangle$. Because of $u \ll\langle\phi\rangle$, the observed neutrinos $\sim \nu_{L}$ gain masses via a type I seesaw, by

$$
\left[m_{\nu}\right]_{\alpha \beta} \simeq-\left[m_{\nu}^{D}\left(m_{\nu}^{R}\right)^{-1}\left(m_{\nu}^{D}\right)^{T}\right]_{\alpha \beta}=h_{\alpha a}^{\nu}\left(h^{R}\right)_{a b}^{-1}\left(h^{\nu}\right)_{b \beta}^{T} \frac{u^{2}}{2\langle\phi\rangle} \sim \frac{u^{2}}{\langle\phi\rangle} .
$$

Fitting the data $m_{\nu} \sim 0.1 \mathrm{eV}$, we obtain $\langle\phi\rangle \sim\left[\left(h^{\nu}\right)^{2} / h^{R}\right] 10^{14} \mathrm{GeV}$, since $u$ is proportional to the weak scale. Given that $h^{\nu}, h^{R} \sim 1$, one has $\langle\phi\rangle \sim 10^{14} \mathrm{GeV}$, close to the grand unification scale. The right-handed neutrinos $\nu_{a R}$ have masses in this scale. 
It is clear that two neutrinos $\nu_{2,3 L}$ achieve masses via the type I seesaw with the corresponding mixing angle $\theta_{23}$ comparable to the data, while the neutrino $\nu_{1 L}$ has a mass (which one sets $h^{\xi} \kappa \sim 0.1 \mathrm{eV}$ ) via the type II seesaw and does not mix with $\nu_{2,3 L}$. The mixing angles $\theta_{12}$ and $\theta_{13}$ can be induced by an effective interaction, such as

$$
\mathcal{L}_{\text {mix }}=\frac{h_{1 \beta}^{\nu}}{\mathcal{M}^{2}} \bar{\psi}_{1 L}^{c} \psi_{\beta L} \rho \eta^{*} \phi+H . c .,
$$

where $\mathcal{M}$ is the new physics scale which can be fixed at $\mathcal{M}=\langle\phi\rangle$. The mass matrix of observed neutrinos is corrected by

$$
\left[m_{\nu}\right]_{1 \beta}=-h_{1 \beta}^{\nu} \frac{u v}{\langle\phi\rangle} \sim \frac{u v}{\langle\phi\rangle},
$$

where $v$ is proportional to the weak scale like $u$. These elements can generate appropriate mixing angles for $\theta_{12,13}$. In this case, the neutrino mass matrix is generic and small.

\subsection{Gauge sector}

The mass Lagrangian of gauge bosons is given by

$$
\mathcal{L} \supset \sum_{\Phi=\eta, \rho, \chi, S}\left(D_{\mu}\langle\Phi\rangle\right)^{\dagger}\left(D^{\mu}\langle\Phi\rangle\right)
$$

where $D_{\mu}=\partial_{\mu}+i g_{s} t_{n} G_{n \mu}+i g T_{n} A_{n \mu}+i g_{X} X B_{\mu}$ is covariant derivative, in which $\left(g_{s}, g, g_{X}\right)$, $\left(t_{n}, T_{n}, X\right)$, and $\left(G_{n}, A_{n}, B\right)$ correspond to the coupling constants, generators, and gauge bosons of the 3-3-1 groups, respectively. ${ }^{2}$ It acts as $D_{\mu}\langle\Phi\rangle=i g\left(T_{n} A_{n \mu}+t_{X} X_{\Phi} B_{\mu}\right)\langle\Phi\rangle$ for a triplet and $D_{\mu}\langle S\rangle=i g\left(T_{n} A_{n \mu}\langle S\rangle+\langle S\rangle T_{n}^{T} A_{n \mu}+t_{X} X_{S} B_{\mu}\langle S\rangle\right)$ for a sextet, where $T_{n}=\frac{1}{2} \lambda_{n}$ are Gell-Mann matrices and $t_{X}=g_{X} / g$.

Define the non-Hermitian gauge bosons,

$$
W^{ \pm}=\frac{1}{\sqrt{2}}\left(A_{1} \mp i A_{2}\right), \quad X^{ \pm}=\frac{1}{\sqrt{2}}\left(A_{4} \mp i A_{5}\right), \quad Y^{0,0 *}=\frac{1}{\sqrt{2}}\left(A_{6} \mp i A_{7}\right) .
$$

They are mass eigenstates by themselves with corresponding masses,

$$
m_{W}^{2} \simeq \frac{g^{2}}{4}\left(u^{2}+v^{2}\right), \quad m_{X}^{2}=\frac{g^{2}}{4}\left(u^{2}+w^{2}+2 \Lambda^{2}\right), \quad m_{Y}^{2} \simeq \frac{g^{2}}{4}\left(v^{2}+w^{2}+2 \Lambda^{2}\right) .
$$

Because of the matter parity conservation, there is no mixing between $W$ and $X$ as well as $A_{6}$ and neutral gauge bosons. Because the type II seesaw requires an infinitesimal $\kappa$, its correction to $m_{W}$ and $m_{Y}$ has been suppressed.

The neutral gauge bosons $\left(A_{3}, A_{8}, B\right)$ mix via a $3 \times 3$ mass matrix. This yields the massless photon field,

$$
A=s_{W} A_{3}+c_{W}\left(\frac{t_{W}}{\sqrt{3}} A_{8}+\sqrt{1-\frac{t_{W}^{2}}{3}} B\right)
$$

\footnotetext{
${ }^{2}$ Note that the $\mathrm{U}(1)_{N}$ part and its scalar were integrated out.
} 
where $s_{W}=e / g=\sqrt{3} t_{X} / \sqrt{3+4 t_{X}^{2}}$ is the sine of the Weinberg's angle [71]. As usual, we define the remaining neutral fields orthogonal to $A$ as

$$
\begin{aligned}
Z & =c_{W} A_{3}-s_{W}\left(\frac{t_{W}}{\sqrt{3}} A_{8}+\sqrt{1-\frac{t_{W}^{2}}{3}} B_{\mu}\right), \\
Z^{\prime} & =\sqrt{1-\frac{t_{W}^{2}}{3}} A_{8}-\frac{t_{W}}{\sqrt{3}} B .
\end{aligned}
$$

In the new basis $\left(A, Z, Z^{\prime}\right)$, there is only $Z-Z^{\prime}$ mixing via a $2 \times 2$ mass matrix, while $A$ is decoupled. This mass matrix yields eigenstates

$$
Z_{1}=c_{\varphi} Z-s_{\varphi} Z^{\prime}, \quad Z_{2}=s_{\varphi} Z+c_{\varphi} Z^{\prime}
$$

with masses

$$
\begin{aligned}
& m_{Z_{1}}^{2} \simeq \frac{g^{2}}{4 c_{W}^{2}}\left(u^{2}+v^{2}\right), \\
& m_{Z_{2}}^{2} \simeq \frac{g^{2}}{4\left(3-t_{W}^{2}\right)}\left[\left(1+t_{W}^{2}\right)^{2} u^{2}+\left(1-t_{W}^{2}\right)^{2} v^{2}+4\left(w^{2}+4 \Lambda^{2}\right)\right],
\end{aligned}
$$

and the mixing angle

$$
t_{2 \varphi} \simeq \frac{\sqrt{3-4 s_{W}^{2}}}{2 c_{W}^{4}} \frac{u^{2}-c_{2 W} v^{2}}{w^{2}+4 \Lambda^{2}} .
$$

Since $\kappa$ is tiny, its contribution to the $\rho$ parameter is neglected. The deviation of the $\rho$ parameter from the standard model prediction is due to the $Z-Z^{\prime}$ mixing, obtained by

$$
\Delta \rho \simeq \frac{\left(u^{2}-c_{2 W} v^{2}\right)^{2}}{4 c_{W}^{4}\left(u^{2}+v^{2}\right)\left(w^{2}+4 \Lambda^{2}\right)} .
$$

From the $W$ mass, we derive $u^{2}+v^{2}=(246 \mathrm{GeV})^{2}$. From the global fit, the PDG Collaboration extracts the $\rho$ deviation as $\Delta \rho=0.00039 \pm 0.00019$, which is $2 \sigma$ above the standard model prediction [72]. We contour $\Delta \rho$ as a function of $u=0-246 \mathrm{GeV}$, since $v$ is related to $u$. Generally for the whole $u$ range, the new physics scales are bounded by $\sqrt{w^{2}+4 \Lambda^{2}} \sim 5-7 \mathrm{TeV}$ [73]. However, there is a regime localized at $u=\sqrt{c_{2 W}} v \simeq 145 \mathrm{GeV}$, where both $\Delta \rho$ and $\varphi$ vanish, i.e. the new physics is always decoupled when $w, \Lambda$ tend to zero. Therefore, we can close the 3-3-1 symmetry at the weak scale in this regime [74, 75].

\section{FCNC}

Because the first lepton generation transforms differently from the last two lepton generations under $\mathrm{SU}(3)_{L}$, there exist FCNCs at the tree-level associated with leptons. Note that the FCNCs conserve quark flavors, in contrast to the ordinary 3-3-1 models.

Indeed, the neutral currents of fermions depend on the Cartan (diagonal) charges $T_{3,8}$ and $X=Q-T_{3}-T_{8} / \sqrt{3}$ as follows

$$
\mathcal{L} \supset \bar{F} i \gamma^{\mu} D_{\mu} F \supset-g \bar{F} \gamma^{\mu}\left[T_{3} A_{3 \mu}+T_{8} A_{8 \mu}+t_{X}\left(Q-T_{3}-T_{8} / \sqrt{3}\right) B_{\mu}\right] F,
$$


where $F$ runs over fermion multiplets. It is easily verified that all the quarks $u_{a L}, u_{a R}, d_{a L}$, $d_{a R}, U_{a L}$, and $U_{a R}$ as well as the right-handed leptons $e_{a R}$ and $E_{a R}$ correspondingly do not flavor-change. Also, the terms of $T_{3}$ and $Q$ conserve all fermion flavors. There remains

$$
\mathcal{L} \supset-g \bar{\psi}_{a L} \gamma^{\mu} T_{8} \psi_{a L}\left(A_{8 \mu}-\frac{t_{X}}{\sqrt{3}} B_{\mu}\right)=-\frac{g}{\sqrt{1-t_{W}^{2} / 3}} \bar{\psi}_{a L} \gamma^{\mu} T_{8} \psi_{a L} Z_{\mu}^{\prime},
$$

where note that $T_{8} \psi_{\alpha L}=\frac{1}{2} \lambda_{8} \psi_{\alpha L}$, while $T_{8} \psi_{1 L}=\frac{1}{2} \lambda_{8} \psi_{1 L}+\psi_{1 L} \frac{1}{2} \lambda_{8}$ and the corresponding interaction is traced. We obtain

$$
\mathcal{L} \supset-\frac{g}{2 \sqrt{3-t_{W}^{2}}}\left(\bar{\nu}_{L} \gamma^{\mu} T_{\nu} \nu_{L}+\bar{e}_{L} \gamma^{\mu} T_{e} e_{L}+\bar{E}_{L} \gamma^{\mu} T_{E} E_{L}\right) Z_{\mu}^{\prime},
$$

where $T_{\nu}=T_{e}=\operatorname{diag}(-1,1,1)$ and $T_{E}=\operatorname{diag}(-4,-2,-2)$. We commonly denote $l=\nu$ or $e$ or $E$ and change to the mass basis $l_{L, R}=V_{l L, R} l_{L, R}^{\prime}$. The relevant Lagrangian is

$$
\mathcal{L} \supset-\frac{g}{2 \sqrt{3-t_{W}^{2}}} \bar{l}_{L}^{\prime} \gamma^{\mu}\left(V_{l L}^{\dagger} T_{l} V_{l L}\right) l_{L}^{\prime} Z_{\mu}^{\prime} \equiv \Gamma_{i j}^{l^{\prime} Z^{\prime}} \bar{l}_{i L}^{\prime} \gamma^{\mu} l_{j L}^{\prime} Z_{\mu}^{\prime},
$$

where

$$
\Gamma_{i j}^{l^{\prime} Z^{\prime}}=-\frac{g}{2 \sqrt{3-t_{W}^{2}}}\left(V_{l L}^{\dagger} T_{l} V_{l L}\right)_{i j}=\frac{g}{\sqrt{3-t_{W}^{2}}}\left(V_{l L}^{*}\right)_{1 i}\left(V_{l L}\right)_{1 j}
$$

which takes the same form for both $l=\nu, e$ and $l=E$ as well as flavor-changes for $i \neq j$.

Let us recall that all quark generations transform universally under the 3-3-1 group, which yields the flavor-conserved current with $Z^{\prime}$ as

$$
\begin{aligned}
\mathcal{L} \supset & -\frac{g\left(2+c_{2 W}\right)}{6 c_{W} \sqrt{1+2 c_{2 W}}}\left(\bar{u}_{L} \gamma^{\mu} u_{L}+\bar{d}_{L} \gamma^{\mu} d_{L}\right) Z_{\mu}^{\prime} \\
& -\frac{g s_{W}^{2}}{3 c_{W} \sqrt{1+2 c_{2 W}}}\left(2 \bar{u}_{R} \gamma^{\mu} u_{R}-\bar{d}_{R} \gamma^{\mu} d_{R}\right) Z_{\mu}^{\prime}
\end{aligned}
$$

where we denote physical eigenstates for up-quarks as $u=(u c t)^{T}$, down-quarks as $d=$ $(d s b)^{T}$, and commonly $q=u$ or $d$ in further investigation.

Integrating out the heavy field, $Z^{\prime}$, from (3.4) and/or (3.6), we obtain an effective Lagrangian that sums over six-dimensional interactions (operators) relevant to the standard 
model fermions at the tree-level, such that

$$
\begin{aligned}
& -\frac{\Gamma_{\alpha \beta}^{l Z^{\prime}} \Gamma_{\gamma \delta}^{l Z^{\prime}}}{m_{Z^{\prime}}^{2}}\left(\bar{l}_{\alpha} \gamma^{\mu} P_{L} l_{\beta}\right)\left(\bar{l}_{\gamma} \gamma_{\mu} P_{L} l_{\delta}\right), \\
& -\frac{\Gamma_{\alpha \beta}^{l Z^{\prime}}}{m_{Z^{\prime}}^{2}}\left(\frac{g s_{W}^{2}}{c_{W} \sqrt{1+2 c_{2 W}}}\right)\left(\bar{l}_{\alpha} \gamma^{\mu} P_{L} l_{\beta}\right)\left(\bar{l}_{\delta} \gamma_{\mu} P_{R} l_{\delta}\right), \\
& -\frac{\Gamma_{\alpha \beta}^{l Z^{\prime}} \Gamma_{\gamma \delta}^{\nu Z^{\prime}}}{m_{Z^{\prime}}^{2}}\left(\bar{\nu}_{\gamma} \gamma_{\mu} P_{L} \nu_{\delta}\right)\left(\bar{l}_{\alpha} \gamma^{\mu} P_{L} l_{\beta}\right), \\
& -\frac{\Gamma_{\alpha \beta}^{\nu Z^{\prime}}}{m_{Z^{\prime}}^{2}}\left(\frac{g s_{W}^{2}}{c_{W} \sqrt{1+2 c_{2 W}}}\right)\left(\bar{\nu}_{\alpha} \gamma_{\mu} P_{L} \nu_{\beta}\right)\left(\bar{l}_{\delta} \gamma^{\mu} P_{R} l_{\delta}\right), \\
& +\frac{\Gamma_{\alpha \beta}^{\nu Z^{\prime}}}{m_{Z^{\prime}}^{2}} \frac{g\left(2+c_{2 W}\right)}{6 c_{W} \sqrt{1+2 c_{2 W}}}\left(\bar{\nu}_{\alpha} \gamma^{\mu} P_{L} \nu_{\beta}\right)\left(\bar{q} \gamma_{\mu} P_{L} q\right), \\
& +\frac{\Gamma_{\alpha \beta}^{\nu Z^{\prime}}}{m_{Z^{\prime}}^{2}} \frac{g s_{W}^{2}}{3 c_{W} \sqrt{1+2 c_{2 W}}}\left(\bar{\nu}_{\alpha} \gamma^{\mu} P_{L} \nu_{\beta}\right)\left(\eta^{q} \bar{q} \gamma_{\mu} P_{R} q\right), \\
& +\frac{\Gamma_{\alpha \beta}^{l Z^{\prime}}}{m_{Z^{\prime}}^{2}} \frac{g\left(2+c_{2 W}\right)}{6 c_{W} \sqrt{1+2 c_{2 W}}}\left(\bar{l}_{\alpha} \gamma^{\mu} P_{L} l_{\beta}\right)\left(\bar{q} \gamma_{\mu} P_{L} q\right), \\
& +\frac{\Gamma_{\alpha \beta}^{l Z^{\prime}}}{m_{Z^{\prime}}^{2}} \frac{g s_{W}^{2}}{3 c_{W} \sqrt{1+2 c_{2 W}}}\left(\bar{l}_{\alpha} \gamma^{\mu} P_{L} l_{\beta}\right)\left(\eta^{q} \bar{q} \gamma_{\mu} P_{R} q\right), \\
& -\frac{1}{m_{Z^{\prime}}^{2}}\left(\frac{g\left(2+c_{2 W}\right)}{6 c_{W} \sqrt{1+2 c_{2 W}}}\right)^{2}\left(\bar{q} \gamma^{\mu} P_{L} q\right)\left(\bar{q} \gamma_{\mu} P_{L} q\right), \\
& -\frac{1}{m_{Z^{\prime}}^{2}}\left(\frac{g s_{W}^{2}}{3 c_{W} \sqrt{1+2 c_{2 W}}}\right)^{2}\left(\eta^{q} \bar{q} \gamma_{\mu} P_{R} q\right)\left(\eta^{q} \bar{q} \gamma_{\mu} P_{R} q\right) .
\end{aligned}
$$

For convenience, we have relabeled the lepton eigenstates $\left(l_{i}^{\prime}\right)$ to be $l_{\alpha}$, which are determined by a Greece (letter) index $\alpha=1,2,3$ and should not be confused with a generation index, such that $e_{\alpha}=e, \mu, \tau$ and $\nu_{\alpha}=\nu_{1}, \nu_{2}, \nu_{3}$. A coefficient $\eta^{q}$ applies for the up-quarks as $\eta^{u}=2$ and the down-quarks as $\eta^{d}=-1$.

The present constraints for the effective Lagrangian come from several processes. The first two terms (3.7) and (3.8) provide charged lepton flavor violating processes like $\mu \rightarrow 3 e$, $\tau \rightarrow 3 e, \tau \rightarrow 3 \mu, \tau \rightarrow 2 e \mu$, and $\tau \rightarrow 2 \mu e$. The next four terms (3.9), (3.10), (3.11), and (3.12) present wrong muon and tau decays as well as the nonstandard neutrino interactions that concern both constraints from oscillation and non-oscillation experiments. The last four terms (3.13), (3.14), (3.15), and (3.16) describe semileptonic $\tau \rightarrow \mu(e)$ decays and $\mu-e$ conversion in nuclei as well as the signals for new physics (dilepton, dijet, etc.) at low energy such as the Tevatron. Therefore, in the following, we consider the phenomenological aspect due to the presence of the interactions given above. 


\section{Phenomenology}

\subsection{Leptonic three-body decays}

To perform the analysis in the current section, we suppose that the major sources contributing to the lepton flavor violating processes come from the direct gauge interactions between the charged leptons and new massive gauge boson $Z^{\prime}$, while the others such as scalar contributions are considered to be small and neglected. The involved interactions, which are fully introduced in the previous section expressed in eqs. (3.7)-(3.16), have the amplitudes inversely proportional to the square of the new gauge boson mass, $m_{Z^{\prime}}^{2}$, as well as dependent on the charged lepton mixing matrix $V_{e L}$. Moreover, the branching ratios or conversing ratio for $\mu \rightarrow e$ conversion in nuclei of the processes of interest are proportional to the square of the lepton-flavor violating effective interaction strengths, thus to be suppressed by the power fourth of the new gauge boson mass $m_{Z^{\prime}}^{4}$.

The 3-by-3 unitary charged lepton mixing matrix $V_{e L}$ is undetermined, although $V_{\nu L}^{\dagger} V_{e L}$ is constrained by the neutrino oscillation data. In the current research, we parametrize $V_{e L}$ using three Euler angles $\left(\theta_{i j}^{\ell}\right)$ and a phase $\left(\delta^{\ell}\right)$, which is similar to the parametrizations of the CKM and PMNS matrices,

$$
V_{e L}=\left(\begin{array}{ccc}
c_{12} c_{13} & s_{12} c_{13} & s_{13} e^{-i \delta^{\ell}} \\
-c_{23} s_{12}-s_{13} s_{23} c_{12} e^{i \delta^{\ell}} & c_{23} c_{12}-s_{13} s_{23} s_{12} e^{i \delta^{\ell}} & s_{23} c_{13} \\
s_{23} s_{12}-s_{13} c_{23} c_{12} e^{i \delta^{\ell}} & -s_{23} c_{12}-s_{13} c_{23} s_{12} e^{i \delta^{\ell}} & c_{23} c_{13}
\end{array}\right) .
$$

Here, we use the notations $c_{i j} \equiv \cos \theta_{i j}^{\ell}$ and $s_{i j} \equiv \sin \theta_{i j}^{\ell}$, and the angles vary in the corresponding ranges, $\theta_{i j}^{\ell}=[0, \pi / 2]$ and $\delta^{\ell}=[0,2 \pi]$.

\subsection{1 $\tau^{+} \rightarrow \mu^{+} \mu^{+} \mu^{-}, \tau^{+} \rightarrow e^{+} e^{+} e^{-}$and $\mu^{+} \rightarrow e^{+} e^{+} e^{-}$}

The lepton flavor violating processes of a lepton decaying into three identical lighter ones, which are usually called as the type I trilepton decays, have been considered before in various models and scenarios of elementary particle physics [76-81]. On the experimental side, the temporary upper bound constraints on the branching ratios of those processes have also been obtained, such as [72]

$$
\begin{aligned}
& \operatorname{Br}(\mu \rightarrow 3 e)<1.0 \times 10^{-12}, \\
& \operatorname{Br}(\tau \rightarrow 3 e)<2.7 \times 10^{-8}, \\
& \operatorname{Br}(\tau \rightarrow 3 \mu)<2.1 \times 10^{-8} .
\end{aligned}
$$

Thus, the upper bound on $\operatorname{Br}(\mu \rightarrow 3 e)$ is about four orders more stringent than the corresponding channels of $\tau$ decays.

The lower bound on the new gauge boson mass $m_{Z^{\prime}}$ comes from the LHC dilepton and dijet searches, which is roughly set to be larger than $4 \mathrm{TeV}$ for the $Z^{\prime}$ couplings analogous to the standard model $Z$ couplings [82, 83]. However, in this model the $Z^{\prime}$ mass takes a smaller bound, say $2.8 \mathrm{TeV}$, as shown below. Because the transferred momentum, whose maximal value is about the $\tau$ mass, is much smaller than $m_{Z^{\prime}}$, the type I trilepton decay branching 
ratios, for instance $\tau^{+} \rightarrow \mu^{+} \mu^{+} \mu^{-}$, can be calculated with a high enough precision using the effective Lagrangian written as follows

$$
\mathcal{L}^{I}=-\frac{4 G_{F}}{\sqrt{2}}\left[g_{L L}^{I}\left(\bar{\tau} \gamma^{\mu} P_{L} \mu\right)\left(\bar{\mu} \gamma_{\mu} P_{L} \mu\right)+g_{L R}^{I}\left(\bar{\tau} \gamma^{\mu} P_{L} \mu\right)\left(\bar{\mu} \gamma_{\mu} P_{R} \mu\right)\right]+\text { H.c. }
$$

where

$$
g_{L L}^{I}=\frac{\sqrt{2} \Gamma_{\tau \mu}^{l Z^{\prime}} \Gamma_{\mu \mu}^{l Z^{\prime}}}{2 G_{F} m_{Z^{\prime}}^{2}}, \quad g_{L R}^{I}=\left(\frac{\sqrt{2} \Gamma_{\tau \mu}^{l Z^{\prime}}}{4 G_{F} m_{Z^{\prime}}^{2}}\right)\left(\frac{g s_{W}^{2}}{c_{W} \sqrt{1+2 c_{2 W}}}\right) .
$$

The branching ratio for this process from eq. (4.5) can be found in [76],

$$
\operatorname{Br}\left(\tau^{+} \rightarrow \mu^{+} \mu^{+} \mu^{-}\right)=\left(\left|g_{L R}^{I}\right|^{2}+2\left|g_{L L}^{I}\right|^{2}\right) \operatorname{Br}\left(\tau^{+} \rightarrow \bar{\nu}_{\tau} e^{+} \nu_{e}\right)
$$

In the same way, the effective Lagrangians and branching ratios for $\tau^{+} \rightarrow e^{+} e^{+} e^{-}$and $\mu^{+} \rightarrow e^{+} e^{+} e^{-}$channels can be derived. That said, their expressions could be obtained from (4.5) and (4.7) by replacing $\mu$ by $e$ for $\tau \rightarrow 3 e$ and $(\tau, \mu)$ by $(\mu, e)$ for $\mu \rightarrow 3 e$, respectively.

Theoretically, to set the upper bound on $\operatorname{Br}\left(\ell \rightarrow 3 \ell^{\prime}\right)$ in the flipped 3-3-1 model, we vary $\theta_{i j}^{\ell}$ in $[0, \pi / 2]$ and $\delta^{\ell}$ in $[0,2 \pi]$. The result is:

$$
\begin{aligned}
& 0 \leq \operatorname{Br}(\mu \rightarrow 3 e) \leq 4.4272 \times 10^{-5}\left(\frac{1 \mathrm{TeV}}{m_{Z^{\prime}}}\right)^{4}, \\
& 0 \leq \operatorname{Br}(\tau \rightarrow 3 e) \leq 7.8892 \times 10^{-6}\left(\frac{1 \mathrm{TeV}}{m_{Z^{\prime}}}\right)^{4}, \\
& 0 \leq \operatorname{Br}(\tau \rightarrow 3 \mu) \leq 7.6989 \times 10^{-6}\left(\frac{1 \mathrm{TeV}}{m_{Z^{\prime}}}\right)^{4} .
\end{aligned}
$$

Here the minimal (maximal) values frequently occur at many relevant values of $\left(\theta_{i j}^{\ell}, \delta^{\ell}\right)$, for example, at $\left(\sin \theta_{12}^{\ell}, \sin \theta_{13}^{\ell}, \sin \theta_{23}^{\ell}, \delta^{\ell}\right)$ :

$$
\begin{array}{lll}
0.7074,0.0000,0.4336,1.04 \pi & (0.1005,1.0000,0.8601,1.70 \pi), \\
0.0000,0.7071,0.2397,0.45 \pi & (0.8498,0.0000,0.0464,0.99 \pi), \\
1.0000,0.7072,0.5033,0.39 \pi & (0.0000,0.0000,0.8082,0.38 \pi),
\end{array}
$$

according to $\operatorname{Br}(\mu \rightarrow 3 e), \operatorname{Br}(\tau \rightarrow 3 e)$, and $\operatorname{Br}(\tau \rightarrow 3 \mu)$, respectively. Indeed, the minimum is easily seen to be at the points that $\Gamma_{\mu e}^{l Z^{\prime}}, \Gamma_{\tau e}^{l Z^{\prime}}$, and $\Gamma_{\tau \mu}^{l Z^{\prime}}$ vanish, respectively.

In figure 1 , we illustrate the dependence of the branching ratio $\operatorname{Br}\left(\ell \rightarrow 3 \ell^{\prime}\right)$ as a single variable function of the new gauge boson mass $m_{Z^{\prime}}$ for $\theta_{12}^{\ell}=\pi / 3, \theta_{13}^{\ell}=\pi / 6, \theta_{23}^{\ell}=\pi / 4$, and $\delta^{\ell}=0$ in the left panel, and for $\sin \theta_{12}^{\ell}=0.9936, \sin \theta_{13}^{\ell}=0.9953, \sin \theta_{23}^{\ell}=0.2324$, $\delta^{\ell}=1.10 \pi$ in the right panel. The currently experimental upper bounds on the $\tau$ decay channels are omitted from the figure because of their much less stringency comparing to those of $\mu$. As shown in figure 1, the branching ratio lines decreasing as $m_{Z^{\prime}}$ increasing are consistent with the fact that they are inversely proportional to $m_{Z^{\prime}}^{4}$, aforementioned. Using the results shown in figure 1 , one obtains lower limits $m_{Z^{\prime}} \geq 65.3,362.1$, and $652.4 \mathrm{TeV}$ for the left panel and $m_{Z^{\prime}} \geq 3.8,20.6$, and $36.5 \mathrm{TeV}$ for the right panel, corresponding to the current upper bound and future sensitivities of the PSI and PSI upgraded experiments, respectively. The latter values quite agree with the current collision bounds on $Z^{\prime}$ mass. 

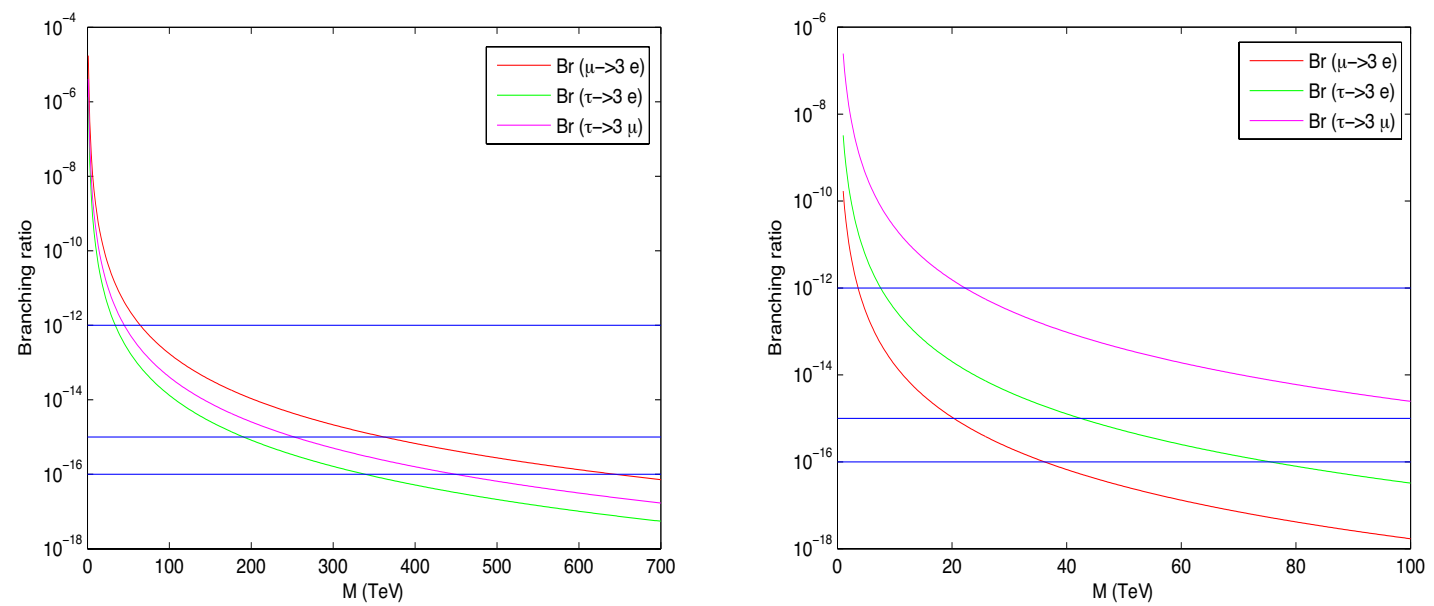

Figure 1. Branching ratios $\operatorname{Br}(\mu \rightarrow 3 e), \operatorname{Br}(\tau \rightarrow 3 e)$, and $\operatorname{Br}(\tau \rightarrow 3 \mu)$ as functions of the new neutral gauge boson mass $m_{Z^{\prime}} \equiv M$, respectively. The three blue horizon-lines correspond to the currently experimental upper bound and expected sensitivities of the PSI and PSI upgraded experiments, namely $\operatorname{Br}(\mu \rightarrow 3 e)=10^{-12}, 10^{-15}$, and $10^{-16}$, respectively. The left-panel is created for $\theta_{12}^{\ell}=\pi / 3, \theta_{13}^{\ell}=\pi / 6, \theta_{23}^{\ell}=\pi / 4$, and $\delta^{\ell}=0$, whereas the right-panel is produced according to $\sin \theta_{12}^{\ell}=0.9936, \sin \theta_{13}^{\ell}=0.9953, \sin \theta_{23}^{\ell}=0.2324$, and $\delta^{\ell}=1.10 \pi$.

\subsection{2 $\tau^{+} \rightarrow \mu^{+} e^{+} e^{-}$and $\tau^{+} \rightarrow e^{+} \mu^{+} \mu^{-}$}

Similar to the decays $\ell \rightarrow 3 \ell^{\prime}$ considered in the previous section, when the new gauge boson mass $m_{Z^{\prime}}$ is at order $\mathrm{TeV}$ or larger, the type II three leptonic decay, e.g. $\tau^{+} \rightarrow \mu^{+} e^{+} e^{-}$, can be well described by an effective Lagrangian, which takes the form

$$
\begin{aligned}
\mathcal{L}^{I I}= & -\frac{4 G_{F}}{\sqrt{2}}\left[g_{L L}^{I I}\left(\bar{\tau} \gamma^{\mu} P_{L} \mu\right)\left(\bar{e} \gamma_{\mu} P_{L} e\right)+g_{L L}^{X}\left(\bar{\tau} \gamma^{\mu} P_{L} e\right)\left(\bar{e} \gamma_{\mu} P_{L} \mu\right)\right. \\
& \left.+g_{L R}^{I I}\left(\bar{\tau} \gamma^{\mu} P_{L} \mu\right)\left(\bar{e} \gamma_{\mu} P_{R} e\right)\right]+H . c .
\end{aligned}
$$

where

$$
g_{L L}^{I I}=\frac{\sqrt{2} \Gamma_{\tau \mu}^{l Z^{\prime}} \Gamma_{e e}^{l Z^{\prime}}}{2 G_{F} m_{Z^{\prime}}^{2}}, \quad g_{L L}^{X}=\frac{\sqrt{2} \Gamma_{\tau e}^{l Z^{\prime}} \Gamma_{e \mu}^{l Z^{\prime}}}{2 G_{F} m_{Z^{\prime}}^{2}}, \quad g_{L R}^{I I}=\left(\frac{\sqrt{2} \Gamma_{\tau \mu}^{l Z^{\prime}}}{4 G_{F} m_{Z^{\prime}}^{2}}\right)\left(\frac{g s_{W}^{2}}{c_{W} \sqrt{1+2 c_{2 W}}}\right) .
$$

Then the branching ratio for $\tau^{+} \rightarrow \mu^{+} e^{+} e^{-}$decay could be expressed as [76],

$$
\operatorname{Br}\left(\tau^{+} \rightarrow \mu^{+} e^{+} e^{-}\right)=\left(\left|g_{L R}^{I I}\right|^{2}+\left|g_{L L}^{I I}\right|^{2}+\left|g_{L L}^{X}\right|^{2}\right) \operatorname{Br}\left(\tau^{+} \rightarrow \bar{\nu}_{\tau} e^{+} \nu_{e}\right) .
$$

With the aid of the interchange symmetry $\mu \leftrightarrow e$, the expression for the branching ratio of the decay $\tau^{+} \rightarrow e^{+} \mu^{+} \mu^{-}$could be easily obtained from the above formula by appropriately replacing $\left(\Gamma_{\tau \mu}^{l Z^{\prime}}, \Gamma_{e e}^{l Z^{\prime}}\right)$ by $\left(\Gamma_{\tau e}^{l Z^{\prime}}, \Gamma_{\mu \mu}^{l Z^{\prime}}\right)$.

Taking into account the same set of values of $\theta_{i j}^{\ell}$ and $\delta^{\ell}$ as in the previous section, we depict in figure 2 the behaviors of the branching ratios, $\tau \rightarrow e \mu \mu$ and $\tau \rightarrow \mu e e$, according to the variation of the new neutral gauge boson mass $m_{Z^{\prime}}$ (green and red lines), respectively. In this figure, the current upper bounds $\operatorname{Br}(\tau \rightarrow e \mu \mu) \leq 2.7 \times 10^{-8}$ and 


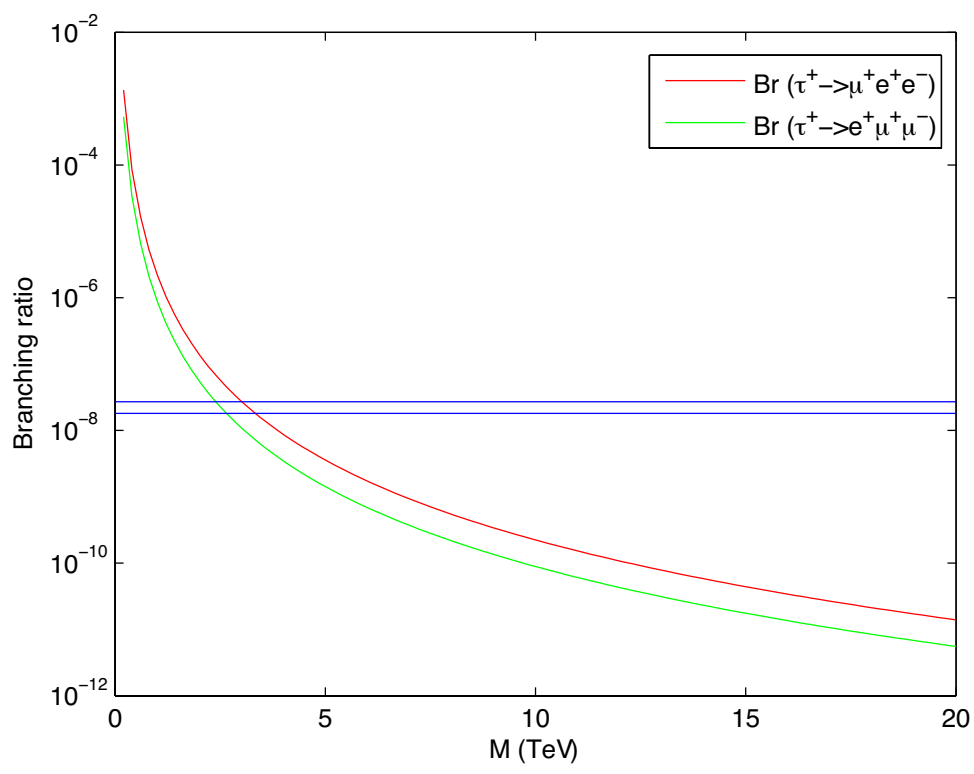

Figure 2. Dependence of the branching ratios $\operatorname{Br}(\tau \rightarrow e \mu \mu)$ and $\operatorname{Br}(\tau \rightarrow \mu e e)$ on the new neutral gauge boson mass, $m_{Z^{\prime}} \equiv M$. Here, the blue lines correspond to the current upper limits $\operatorname{Br}(\tau \rightarrow$ $e \mu \mu) \leq 2.7 \times 10^{-8}$ and $\operatorname{Br}(\tau \rightarrow \mu e e) \leq 1.8 \times 10^{-8}$, respectively.

$\operatorname{Br}(\tau \rightarrow \mu e e) \leq 1.8 \times 10^{-8}$ are also shown as blue lines [72]. The lower limits for $m_{Z^{\prime}}$ obtained from figure 2 responsible for these two processes are roughly $3 \mathrm{TeV}$. This agrees with the limit from $\mu \rightarrow 3 e$ in the case of figure 1 right panel, but is about 20 times less stringent than the limit from $\mu \rightarrow 3 e$ in the case of figure 1 left panel. Particularly, using the current lower bound from $\mu \rightarrow 3 e$ in the latter case, i.e. $m_{Z^{\prime}} \geq 65.3 \mathrm{GeV}$ at $\theta_{12}^{\ell}=\pi / 3$, $\theta_{13}^{\ell}=\pi / 6, \theta_{23}^{\ell}=\pi / 4$, and $\delta^{\ell}=0$, the precision calculation shows that

$$
\begin{aligned}
& \operatorname{Br}(\tau \rightarrow \mu e e) \leq 4.88 \times 10^{-14}, \\
& \operatorname{Br}(\tau \rightarrow e \mu \mu) \leq 1.31 \times 10^{-13} .
\end{aligned}
$$

One the other hand, with the same strategy as in the previous case when varying $\theta_{i j}^{\ell}$ in $[0, \pi / 2]$ and $\delta^{\ell}$ in $[0,2 \pi]$, the bounds derived are

$$
\begin{aligned}
& 0 \leq \operatorname{Br}(\tau \rightarrow \mu e e) \leq 4.4562 \times 10^{-6}\left(\frac{1 \mathrm{TeV}}{m_{Z^{\prime}}}\right)^{4}, \\
& 0 \leq \operatorname{Br}(\tau \rightarrow e \mu \mu) \leq 4.4562 \times 10^{-6}\left(\frac{1 \mathrm{TeV}}{m_{Z^{\prime}}}\right)^{4} .
\end{aligned}
$$

\subsection{3 $\tau^{+} \rightarrow \mu^{+} \mu^{+} e^{-}$and $\tau^{+} \rightarrow e^{+} e^{+} \mu^{-}$}

In this part of search, we consider the type III leptonic decay modes in which lepton flavors are violated by three units, such as $\tau^{+} \rightarrow \mu^{+} \mu^{+} e^{-}$. In the flipped 3-3-1 model, the process is dominantly contributed by an unique effective interaction, which is expressed as

$$
\mathcal{L}^{I I I}=-\frac{4 G_{F}}{\sqrt{2}}\left[g_{L L}^{I I I}\left(\bar{\tau} \gamma^{\mu} P_{L} \mu\right)\left(\bar{e} \gamma_{\mu} P_{L} \mu\right)\right]+H . c .
$$




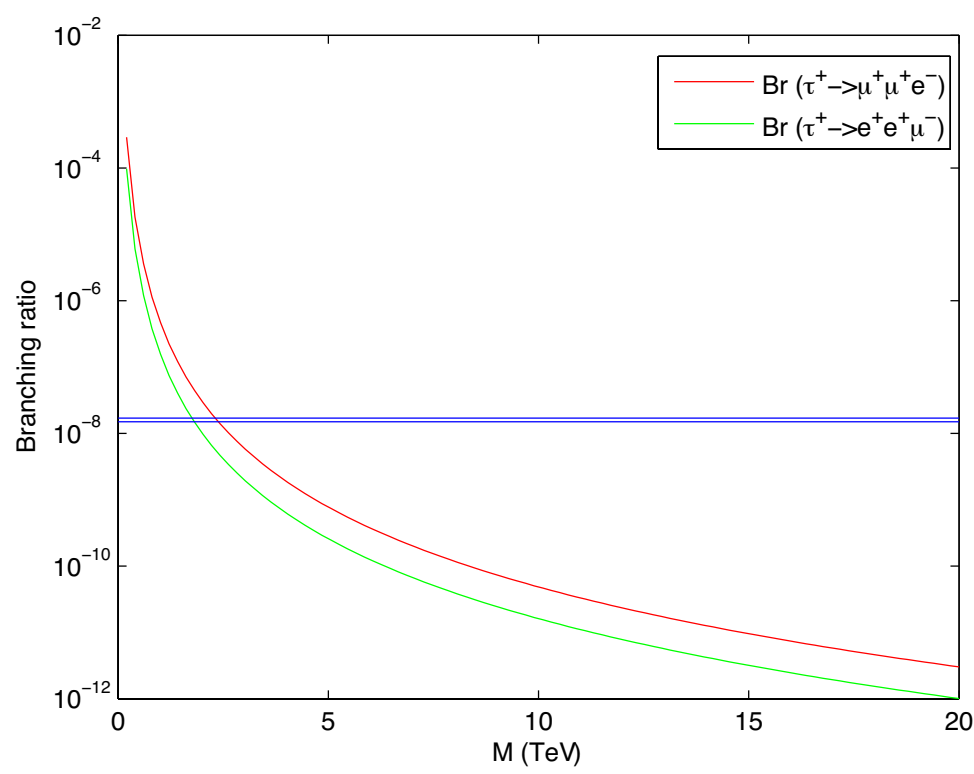

Figure 3. Dependence of the branching ratios $\operatorname{Br}(\tau \rightarrow \mu \mu e)$ and $\operatorname{Br}(\tau \rightarrow e \mu \mu)$ on the new neutral gauge boson mass $m_{Z^{\prime}} \equiv M$. The blue lines correspond to the current upper limits $\operatorname{Br}(\tau \rightarrow \mu \mu e) \leq$ $1.7 \times 10^{-8}$ and $\operatorname{Br}(\tau \rightarrow e e \mu) \leq 1.5 \times 10^{-8}$, which are almost coincided [72].

where

$$
g_{L L}^{I I I}=\frac{\sqrt{2} \Gamma_{\tau \mu}^{l Z^{\prime}} \Gamma_{e \mu}^{l Z^{\prime}}}{2 G_{F} m_{Z^{\prime}}^{2}} .
$$

Then, the branching ratio for $\tau^{+} \rightarrow \mu^{+} \mu^{+} e^{-}$could be easily written down by generalizing the results given in [76] as

$$
\operatorname{Br}\left(\tau^{+} \rightarrow \mu^{+} \mu^{+} e^{-}\right)=2\left|g_{L L}^{I I I}\right|^{2} \operatorname{Br}\left(\tau^{+} \rightarrow \bar{\nu}_{\tau} e^{+} \nu_{e}\right) .
$$

On the other hand, the effective Lagrangian and branching ratio for $\operatorname{Br}\left(\tau^{+} \rightarrow e^{+} e^{+} \mu^{-}\right)$ can be achieved from (4.21) and (4.23) simply by interchanging the fields $\mu \leftrightarrow e$.

Currently, the experimental constraints on the branching ratios of the decays $\tau^{+} \rightarrow$ $\mu^{+} \mu^{+} e^{-}$and $\tau^{+} \rightarrow e^{+} e^{+} \mu^{-}$are, indeed, very weak. Therefore, the corresponding lower limits on the new neutral gauge boson mass $m_{Z^{\prime}}$ given by these two channels are less substantial than those obtained from the channels considered above, especially for the $\mu \rightarrow 3 e$ decay. To be concrete, the behaviors of the $\tau^{+} \rightarrow \mu^{+} \mu^{+} e^{-}$and $\tau^{+} \rightarrow e^{+} e^{+} \mu^{-}$ branching ratios can be found in figure 3 . Using the same trick as in the previous case, we obtain the theoretical upper bounds as follows

$$
\begin{aligned}
& \operatorname{Br}(\tau \rightarrow \mu \mu e) \leq 3.20 \times 10^{-14}, \\
& \operatorname{Br}(\tau \rightarrow e e \mu) \leq 8.17 \times 10^{-15},
\end{aligned}
$$

for $\theta_{12}^{\ell}=\pi / 3, \theta_{13}^{\ell}=\pi / 6, \theta_{23}^{\ell}=\pi / 4$, and $\delta^{\ell}=0$. Hence, these bounds are about six orders below the sensitivities of the current experiments. 
Last, but not least, varying the mixing angles and phase in the allowed regimes, the specified ranges for the $\tau^{+} \rightarrow \mu^{+} \mu^{+} e^{-}$and $\tau^{+} \rightarrow e^{+} e^{+} \mu^{-}$branching ratios are found as

$$
\begin{aligned}
& 0 \leq \operatorname{Br}(\tau \rightarrow \mu \mu e) \leq 5.1155 \times 10^{-7}\left(\frac{1 \mathrm{TeV}}{m_{Z^{\prime}}}\right)^{4}, \\
& 0 \leq \operatorname{Br}(\tau \rightarrow e e \mu) \leq 5.1155 \times 10^{-7}\left(\frac{1 \mathrm{TeV}}{m_{Z^{\prime}}}\right)^{4} .
\end{aligned}
$$

\subsubsection{Comment on wrong $\mu$ and $\tau$ decays}

It is not hard to point out that the wrong muon and tau decays, e.g. $\mu \rightarrow e \nu_{e} \bar{\nu}_{\mu}$ and $\tau \rightarrow \mu \nu_{\mu} \bar{\nu}_{\tau}$, take the same rate as of those in the previous section, respectively. Hence, such decays are far below the experimental limits $\mathrm{Br} \sim 0.1$ [72].

\subsection{Semileptonic $\tau \rightarrow \mu$ and $\tau \rightarrow e$ decays}

The next topic we discuss in this paper is the semileptonic decays of $\tau$, say

$$
\operatorname{Br}\left(\tau^{+} \rightarrow \ell^{+} P\right) \text { and } \operatorname{Br}\left(\tau^{+} \rightarrow \ell^{+} V\right)
$$

in which $\ell=e, \mu$ and $P, V$ stand for neutral pseudoscalar and vector mesons, respectively. These decay channels have been studied formerly in the other models as well as modelindependent scenarios $[76,84,85]$. In the present model, these processes happen dominantly through the exchange of the new neutral gauge boson with lepton-flavor violating interactions. On the experimental side, the following upper bounds have been obtained [72]:

$$
\begin{aligned}
\operatorname{Br}\left(\tau^{+} \rightarrow e^{+} \pi^{0}\right)<8.0 \times 10^{-8}, & & \operatorname{Br}\left(\tau^{+} \rightarrow \mu^{+} \pi^{0}\right)<1.1 \times 10^{-7}, \\
\operatorname{Br}\left(\tau^{+} \rightarrow e^{+} \eta^{0}\right)<9.2 \times 10^{-8}, & & \operatorname{Br}\left(\tau^{+} \rightarrow \mu^{+} \eta^{0}\right)<6.5 \times 10^{-8}, \\
\operatorname{Br}\left(\tau^{+} \rightarrow e^{+} \eta^{\prime 0}\right)<1.6 \times 10^{-7}, & & \operatorname{Br}\left(\tau^{+} \rightarrow \mu^{+} \eta^{0}\right)<1.3 \times 10^{-7}, \\
\operatorname{Br}\left(\tau^{+} \rightarrow e^{+} \rho^{0}\right)<1.8 \times 10^{-8}, & & \operatorname{Br}\left(\tau^{+} \rightarrow \mu^{+} \rho^{0}\right)<1.2 \times 10^{-8}, \\
\operatorname{Br}\left(\tau^{+} \rightarrow e^{+} \omega^{0}\right)<4.8 \times 10^{-8}, & & \operatorname{Br}\left(\tau^{+} \rightarrow \mu^{+} \omega^{0}\right)<4.7 \times 10^{-8}, \\
\operatorname{Br}\left(\tau^{+} \rightarrow e^{+} \phi^{0}\right)<3.1 \times 10^{-8}, & & \operatorname{Br}\left(\tau^{+} \rightarrow \mu^{+} \phi^{0}\right)<8.4 \times 10^{-8},
\end{aligned}
$$

The near future and planned experiments such as LCHb [86], BESIII [87], Belle II [88], and COMET [89] will improve upon these present limits.

As already supplied in (3.13) and (3.14), the effective Lagrangian for the semileptonic decays of the $\tau \rightarrow \mu(e)$ types can be rewritten in the form

$$
\mathcal{L}_{H a d}^{I}=-\frac{4 G_{F}}{\sqrt{2}}\left[h_{L L}^{q \ell}\left(\bar{\tau} \gamma^{\mu} P_{L} \ell\right)\left(\bar{q} \gamma_{\mu} P_{L} q\right)+h_{L R}^{q \ell}\left(\bar{\tau} \gamma^{\mu} P_{L} \ell\right)\left(\bar{q} \gamma_{\mu} P_{R} q\right)\right]+\text { H.c. }
$$

where $\ell=\mu$ or $e$ and the couplings are

$$
h_{L L}^{q \ell}=\frac{g \sqrt{2}\left(2+c_{2 W}\right) \Gamma_{\tau \ell}^{l Z^{\prime}}}{24 G_{F} m_{Z^{\prime}}^{2} c_{W} \sqrt{1+2 c_{2 W}}}, \quad h_{L R}^{q \ell}=\frac{g \sqrt{2} s_{W}^{2} \Gamma_{\tau \ell}^{l Z^{\prime}} \eta_{q}}{12 G_{F} m_{Z^{\prime}}^{2} c_{W} \sqrt{1+2 c_{2 W}}} .
$$


The branching ratio for the $\tau^{+} \rightarrow \ell^{+} P$ decay, where $P$ denotes a neutral pseudoscalar meson as $\pi^{0}$ or $\eta^{0}$ or $\eta^{\prime 0}$, can be derived from (4.30) as follows [76]

$$
\operatorname{Br}\left(\tau^{+} \rightarrow \ell^{+} P\right)=\tau_{\tau} \frac{G_{F}^{2} m_{\tau}^{3}}{4 \pi}\left(1-\frac{m_{P}^{2}}{m_{\tau}^{2}}\right)\left|C_{\text {eff }}^{\ell}\right|^{2},
$$

where $\tau_{\tau}$ is the tau's lifetime and

$$
C_{\mathrm{eff}}^{\ell}=\frac{f_{\eta, \eta^{\prime}}^{q}}{2 \sqrt{2}}\left(h_{L R}^{u \ell}+h_{L R}^{d \ell}-h_{L L}^{u \ell}-h_{L L}^{d \ell}\right)+\frac{f_{\eta, \eta^{\prime}}^{s}}{2}\left(h_{L R}^{d \ell}-h_{L L}^{d \ell}\right),
$$

for $P=\eta^{0}$ or $P=\eta^{\prime 0}$, and

$$
C_{\mathrm{eff}}^{\ell}=\frac{f_{\pi}}{2 \sqrt{2}}\left(h_{L R}^{u \ell}-h_{L R}^{d \ell}-h_{L L}^{u \ell}+h_{L L}^{d \ell}\right)
$$

for $P=\pi^{0}$. Here the decay constant $f_{\pi}=135 \mathrm{MeV}$, while $f_{\eta, \eta^{\prime}}^{q}$ and $f_{\eta, \eta^{\prime}}^{s}$ are defined as

$$
\left(\begin{array}{cc}
f_{\eta}^{q} & f_{\eta}^{s} \\
f_{\eta}^{\prime q} & f_{\eta}^{\prime s}
\end{array}\right)=\left(\begin{array}{cc}
f_{q} \cos \phi_{\eta} & -f_{s} \sin \phi_{\eta} \\
f_{q} \sin \phi_{\eta} & f_{s} \cos \phi_{\eta}
\end{array}\right),
$$

with $f_{q} \simeq 1.07 f_{\pi}, f_{s} \simeq 1.34 f_{\pi}$ and $\phi_{\eta} \simeq 0.2183 \pi$.

Based on the current experimental limits, our numerical calculation will show that the branching ratios of semileptonic $\tau$ decays into the pseudoscalar mesons are actually small to impose meaningful constraints on the relevant parameters in the flipped 3-3-1 model. For concreteness, the results of varying $\theta_{i j}^{\ell}$ and $\delta^{\ell}$ in the allowed ranges lead to

$$
\begin{aligned}
& 0 \leq \operatorname{Br}(\tau \rightarrow e(\mu) \pi) \leq 9.6517 \times 10^{-8}\left(\frac{1 \mathrm{TeV}}{m_{Z^{\prime}}}\right)^{4}, \\
& 0 \leq \operatorname{Br}(\tau \rightarrow e(\mu) \eta) \leq 2.3196 \times 10^{-6}\left(\frac{1 \mathrm{TeV}}{m_{Z^{\prime}}}\right)^{4}, \\
& 0 \leq \operatorname{Br}\left(\tau \rightarrow e(\mu) \eta^{\prime}\right) \leq 2.0475 \times 10^{-6}\left(\frac{1 \mathrm{TeV}}{m_{Z^{\prime}}}\right)^{4} .
\end{aligned}
$$

Let us note that, although the maximal values of the branching ratios obtained above do not depend on the lepton types, namely $\operatorname{Max}[\operatorname{Br}(\tau \rightarrow e P)]=\operatorname{Max}[\operatorname{Br}(\tau \rightarrow \mu P)]$ for any given $P$, they reach, however, the maximal values for different sets of lepton mixing parameters. For instance, $\operatorname{Br}(\tau \rightarrow e \pi)$ has the maximal value at $\sin \theta_{12}^{\ell}=0.0000, \sin \theta_{13}^{\ell}=$ $0.7072, \sin \theta_{23}^{\ell}=0.7295$, and $\delta^{\ell}=1.13 \pi$, whereas $\operatorname{Br}(\tau \rightarrow e \eta)$ gets the maximal value when $\sin \theta_{12}^{\ell}=1.0000, \sin \theta_{13}^{\ell}=0.7073, \sin \theta_{23}^{\ell}=0.8195$, and $\delta^{\ell}=1.92 \pi$.

The theoretical maximal values given in (4.36) are roundly the same orders as the current experimental limits [cf. (4.29)]. Thus the constraints on the new neutral gauge boson mass derived from these channels have much appealing, validating the model. This can be more visually seen in figure 4, where we show the dependence of the branching ratios $\operatorname{Br}\left(\tau^{+} \rightarrow \ell^{+} P\right)$ on the new neutral gauge boson mass $m_{Z^{\prime}} \equiv M$ for $\ell=e, \mu$ and $P=\pi, \eta, \eta^{\prime}$. Since the current upper bounds on the branching ratios are around $10^{-7}$, the 


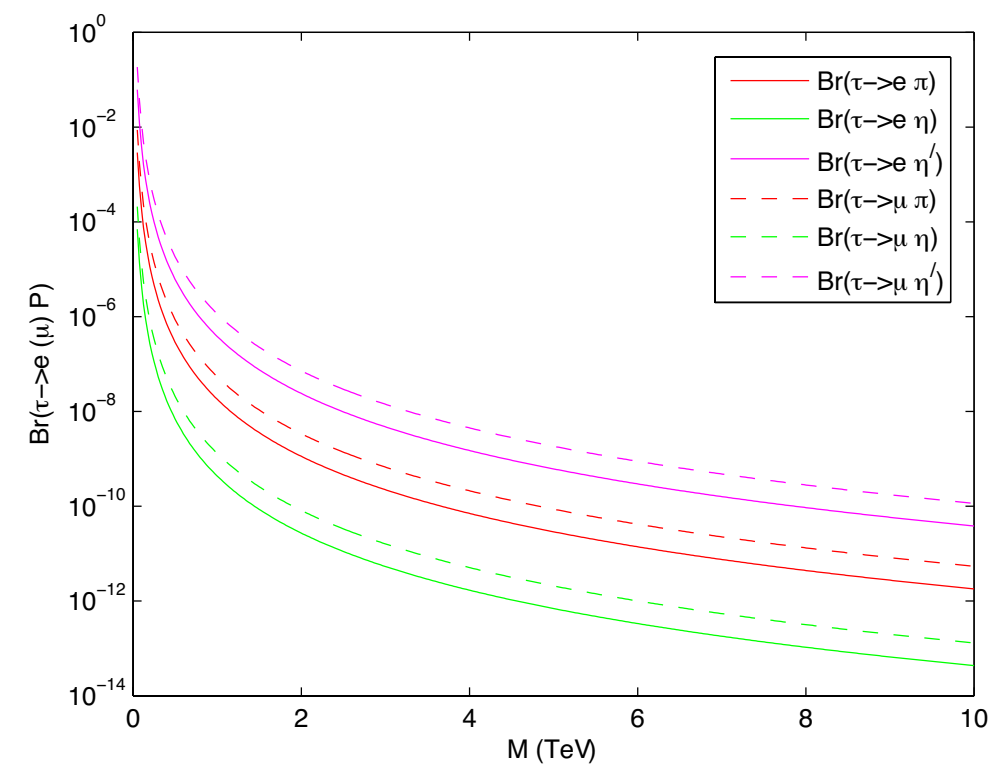

Figure 4. Dependence of the branching ratios $\operatorname{Br}\left(\tau^{+} \rightarrow \ell^{+} P\right)$ on the new neutral gauge boson mass $m_{Z^{\prime}} \equiv M$, where $\ell=e, \mu$ and $P=\pi, \eta, \eta^{\prime}$. Here, lepton mixing angles and phase $\theta_{12}^{\ell}=\pi / 3$, $\theta_{13}^{\ell}=\pi / 6, \theta_{23}^{\ell}=\pi / 4$, and $\delta^{\ell}=0$ have been used.

lower limit obtained for the new neutral gauge boson mass $m_{Z^{\prime}}$ is about $3 \mathrm{TeV}$, which is the same limit set by the searches of the LHC dilepton and dijet signals.

Similar conclusions are also obtained for the case of $\tau^{+} \rightarrow \ell^{+} V$ decay, where $V$ is taken as a neutral vector meson $\rho$ or $\omega$ or $\phi$. The branching ratios for these processes can be derived from the Lagrangian (4.30) as [76]

$$
B r\left(\tau^{+} \rightarrow \ell^{+} V\right)=\tau_{\tau} \frac{G_{F}^{2} m_{\tau}^{3}}{\pi}\left(1-\frac{m_{V}^{2}}{m_{\tau}^{2}}\right)^{2}\left(\frac{m_{\tau}^{2}+2 m_{V}^{2}}{4 m_{V}^{2}}\right)\left|G_{L V}^{\ell}\right|^{2}
$$

where $G_{L V}^{\ell}$ is an effective coupling that takes one of the following forms,

- $G_{L V}^{\ell}=\frac{f_{\rho} m_{\rho}}{2 \sqrt{2} m_{\tau}}\left(h_{L L}^{u \ell}-h_{L L}^{d \ell}+h_{L R}^{u \ell}-h_{L R}^{d \ell}\right)$ for $V=\rho$.

- $G_{L V}^{\ell}=\frac{f_{\omega} m_{\omega}}{2 \sqrt{2} m_{\tau}}\left(h_{L L}^{u \ell}+h_{L L}^{d \ell}+h_{L R}^{u \ell}+h_{L R}^{d \ell}\right)$ for $V=\omega$.

- $G_{L V}^{\ell}=-\frac{f_{\phi} m_{\phi}}{2 m_{\tau}}\left(h_{L L}^{s \ell}+h_{L R}^{s \ell}\right)$ for $V=\phi$.

The coefficients $f_{\rho}=221 \mathrm{MeV}, f_{\omega}=196 \mathrm{MeV}$, and $f_{\phi}=228 \mathrm{MeV}$ are the form factors of the neutral vector mesons $\rho, \omega$, and $\phi$, respectively. 


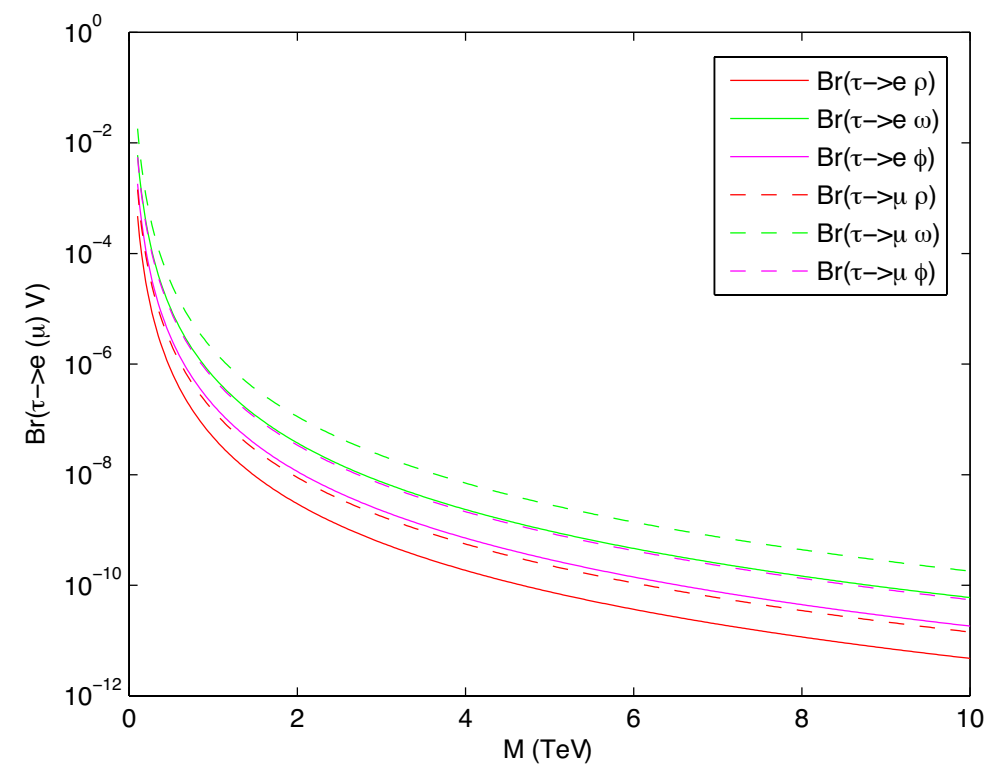

Figure 5. Dependence of the branching ratios $\operatorname{Br}\left(\tau^{+} \rightarrow \ell^{+} V\right)$ on the new neutral gauge boson mass $m_{Z^{\prime}} \equiv M$, where $\ell=e, \mu$ and $P=\rho, \omega, \phi$. To produce the figure, lepton mixing angles and phase $\theta_{12}^{\ell}=\pi / 3, \theta_{13}^{\ell}=\pi / 6, \theta_{23}^{\ell}=\pi / 4$, and $\delta^{\ell}=0$ have been taken.

The identified ranges of the branching ratios when varying the parameters $\theta_{i j}^{\ell}$ and $\delta^{\ell}$ in their domains are

$$
\begin{aligned}
& 0 \leq \operatorname{Br}(\tau \rightarrow e(\mu) \rho) \leq 2.5390 \times 10^{-7}\left(\frac{1 \mathrm{TeV}}{m_{Z^{\prime}}}\right)^{4}, \\
& 0 \leq \operatorname{Br}(\tau \rightarrow e(\mu) \omega) \leq 3.2013 \times 10^{-6}\left(\frac{1 \mathrm{TeV}}{m_{Z^{\prime}}}\right)^{4}, \\
& 0 \leq \operatorname{Br}(\tau \rightarrow e(\mu) \phi) \leq 9.4756 \times 10^{-7}\left(\frac{1 \mathrm{TeV}}{m_{Z^{\prime}}}\right)^{4} .
\end{aligned}
$$

The detail behaviors of the branching ratios $\operatorname{Br}\left(\tau^{+} \rightarrow \ell^{+} V\right)$ are depicted in figure 5 for $\theta_{12}^{\ell}=\pi / 3, \theta_{13}^{\ell}=\pi / 6, \theta_{23}^{\ell}=\pi / 4$, and $\delta^{\ell}=0$. Comparing to the experimental bounds yields a $Z^{\prime}$ mass around $3 \mathrm{TeV}$.

\section{3 $\mu-e$ conversion in nuclei}

In this sector, we consider a hypothetical process, called $\mu \rightarrow e$ conversion in nuclei, in which negative muons are captured in a target of atomic nuclei, such as Titanium (Ti), Aluminum ( $\mathrm{Al})$ or Gold $(\mathrm{Au})$, to form muonic atoms. The muon then converses into an electron in the nuclear field without creating a neutrino. There are a number of experiments that have been built or planned to built to search for the process's signals, for instance TRIUMP [90], SINDRUM-II [91], and COMET [92]. The current experimental limits on the branching ratios are $4.3 \times 10^{-12}$ at TRIUMF for Titanium target and $7 \times 10^{-13}$ for Gold target by SINDRUM-II. Furthermore, the goal of the future experiment COMET is to probe $\mu-e$ conversion signals with sensitivity about $10^{-16}$. 


\begin{tabular}{|cccc|}
\hline $\mathcal{N}$ & $V^{(p)} m_{\mu}^{-5 / 2}$ & $V^{(n)} m_{\mu}^{-5 / 2}$ & $\omega_{\text {capt }}\left(10^{6} \mathrm{~s}^{-1}\right)$ \\
\hline${ }_{22}^{48} \mathrm{Ti}$ & 0.0396 & 0.0468 & 2.590 \\
${ }_{13}^{27} \mathrm{Al}$ & 0.0161 & 0.0173 & 0.7054 \\
${ }_{79}^{197} \mathrm{Au}$ & 0.0974 & 0.146 & 13.07 \\
${ }_{82}^{208} \mathrm{~Pb}$ & 0.0834 & 0.128 & 13.45 \\
\hline
\end{tabular}

Table 3. Nuclear parameters related to $\mu-e$ conversion in ${ }_{22}^{48} \mathrm{Ti},{ }_{13}^{27} \mathrm{Al},{ }_{79}^{197} \mathrm{Au}$ and ${ }_{82}^{208} \mathrm{~Pb}$.

In the considering model, the $\mu-e$ conversing ratio can be calculated from a Lagrangian, which has same form as (4.30)

$$
\mathcal{L}_{\mu-e}^{I}=-\frac{4 G_{F}}{\sqrt{2}}\left[h_{L L}^{q}\left(\bar{\mu} \gamma^{\mu} P_{L} e\right)\left(\bar{q} \gamma_{\mu} P_{L} q\right)+h_{L R}^{q}\left(\bar{\mu} \gamma^{\mu} P_{L} e\right)\left(\bar{q} \gamma_{\mu} P_{R} q\right)\right]+H . c .
$$

in which

$$
h_{L L}^{q}=\frac{g \sqrt{2}\left(2+c_{2 W}\right) \Gamma_{\mu e}^{l Z^{\prime}}}{24 G_{F} m_{Z^{\prime}}^{2} c_{W} \sqrt{1+2 c_{2 W}}}, \quad h_{L R}^{q}=\frac{g \sqrt{2} s_{W}^{2} \Gamma_{\mu e}^{l Z^{\prime}} \eta_{q}}{12 G_{F} m_{Z^{\prime}}^{2} c_{W} \sqrt{1+2 c_{2 W}}}
$$

The conversing ratio (CR), which is obtained after normalizing to the total nuclear capture rate $\omega_{\text {capt }}$, can be simply expressed as

$$
\begin{aligned}
& \mathrm{CR}\left(\mu^{-} A \rightarrow e^{-} A\right)=\frac{4 G_{F}^{2}}{\omega_{\text {capt }}} \mid\left(2 h_{L L}^{u}+2 h_{L R}^{u}+h_{L L}^{d}+h_{L R}^{d}\right) V^{(p)} \\
&+\left.\left(h_{L L}^{u}+h_{L R}^{u}+2 h_{L L}^{d}+2 h_{L R}^{d}\right) V^{(n)}\right|^{2},
\end{aligned}
$$

where $V^{(n)}$ and $V^{(p)}$ are the overlap integrals and $\omega_{\text {capt }}$ is the total capture rate. For the cases of ${ }_{22}^{48} \mathrm{Ti},{ }_{13}^{27} \mathrm{Al},{ }_{79}^{197} \mathrm{Au}$, and ${ }_{82}^{208} \mathrm{~Pb}$ nuclei, they are given in table 3 [93]. We depict in figure 6 the $\mu-e$ conversion ratios in the nuclei of Titanium, Aluminum, and Gold as functions of the new gauge boson mass $m_{Z^{\prime}}$ for the same sets of parameter values, $\theta_{12}^{\ell}=\pi / 3, \theta_{13}^{\ell}=\pi / 6, \theta_{23}^{\ell}=\pi / 4, \delta^{\ell}=0$ (left panel) and $\sin \theta_{12}^{\ell}=0.9936, \sin \theta_{13}^{\ell}=0.9953$, $\sin \theta_{23}^{\ell}=0.2324, \delta^{\ell}=1.10 \pi$ (right panel), used before concerning figure 1 . The present upper limits give stronger constraints on the new neutral gauge boson mass comparing with the other lepton-flavor violating processes considered before. For the first value set of $\left(\theta^{\ell}, \delta^{\ell}\right)$, consistency with the experimental results yields $m_{Z^{\prime}} \geq 116.7 \mathrm{TeV}$ carried with Titanium target and $m_{Z^{\prime}} \geq 204.5 \mathrm{TeV}$ with Gold target, respectively. Moreover, the planned experiment COMMET with sensitivity $10^{-16}$ is possible to probe the conversion signals as long as the new gauge boson is not heavier than about $1468.9 \mathrm{TeV}$. If the lepton mixing parameters are taken as the second set, three lower/upper bounds obtained above are replaced by $m_{Z^{\prime}} \geq 6.7,11.7$, and $84.1 \mathrm{TeV}$. This second cause quite agrees with the collider searches.

Finally, we introduce here the viable regions of the branching ratios for the cases of Titanium, Aluminum, and Gold when varying the lepton mixing parameters in the allowed 

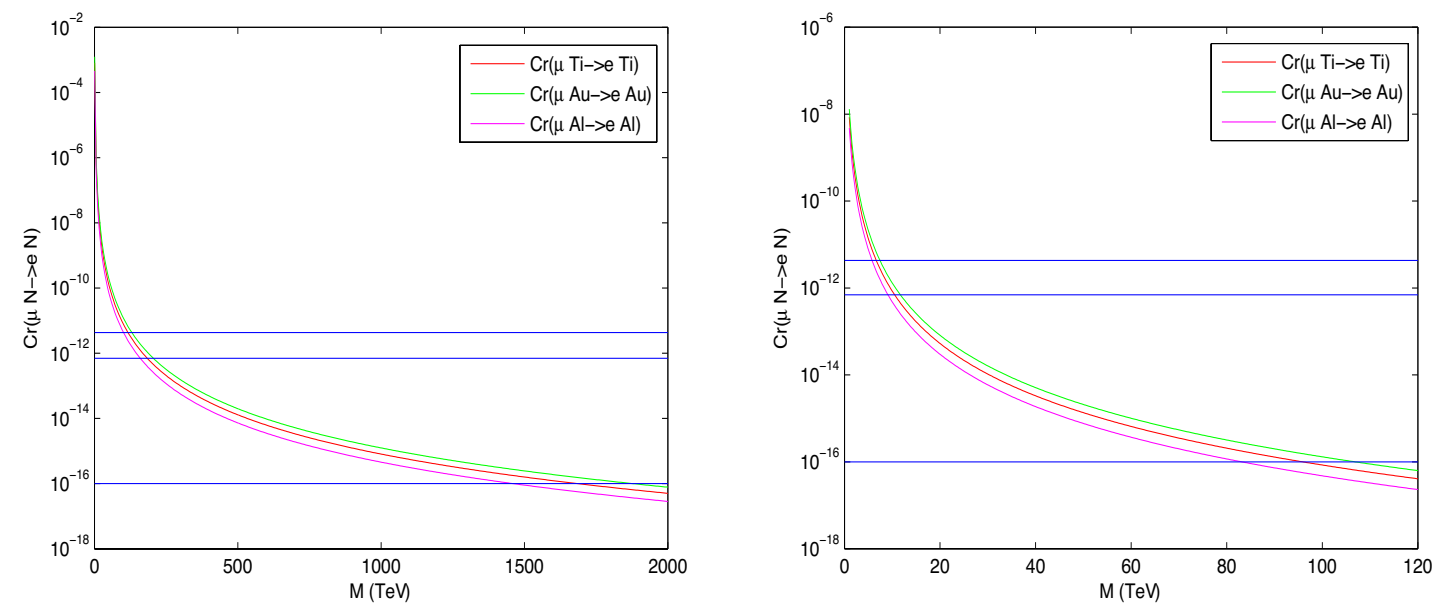

Figure 6. The $\mu \rightarrow e$ conversing ratio $\operatorname{Br}(\mu N \rightarrow e N)$ versus the new neutral gauge boson mass $m_{Z^{\prime}} \equiv M$, for different nuclei: i) ${ }_{22}^{48} \mathrm{Ti}$ (red line), ii) ${ }_{12}^{27} \mathrm{Al}$ (magenta line), and iii) ${ }_{79}^{197} \mathrm{Au}$ (green line). The three blue lines corresponding to $\operatorname{Br}(\mu T i \rightarrow e T i) \leq 4.3 \times 10^{-12}$ [90], $\operatorname{Br}(\mu A u \rightarrow e A u) \leq$ $7.0 \times 10^{-13}$, and $\operatorname{Br}(\mu A l \rightarrow e A l) \leq 1.0 \times 10^{-16}$ are the SINDRUM-II current upper bounds [91] and the COMET expected sentisitvity [92], respectively. The left panel is created with $\theta_{12}^{\ell}=\pi / 3$, $\theta_{13}^{\ell}=\pi / 6, \theta_{23}^{\ell}=\pi / 4$, whereas the right panel is produced for $\sin \theta_{12}^{\ell}=0.9936, \sin \theta_{13}^{\ell}=0.9953$, $\sin \theta_{23}^{\ell}=0.2324, \delta^{\ell}=1.10 \pi$.

ranges, $\theta_{i j}^{\ell}$ in $[0, \pi / 2]$ and $\delta$ in $[0,2 \pi]$ :

$$
\begin{aligned}
& 0 \leq \mathrm{Br}(\mu \mathrm{Ti} \rightarrow e \mathrm{Ti}) \leq 1.9 \times 10^{-3}\left(\frac{1 \mathrm{TeV}}{m_{Z^{\prime}}}\right)^{4} \\
& 0 \leq \operatorname{Br}(\mu \mathrm{Au} \rightarrow e \mathrm{Au}) \leq 3.0 \times 10^{-3}\left(\frac{1 \mathrm{TeV}}{m_{Z^{\prime}}}\right)^{4} \\
& 0 \leq \operatorname{Br}(\mu \mathrm{Al} \rightarrow e \mathrm{Al}) \leq 1.1 \times 10^{-3}\left(\frac{1 \mathrm{TeV}}{m_{Z^{\prime}}}\right)^{4}
\end{aligned}
$$

Here the maximal value occurs at $\left(\sin \theta_{12}^{\ell}, \sin \theta_{13}^{\ell}, \sin \theta_{23}^{\ell}, \delta^{\ell}\right)=$ $(0.7072,0.000,0.8614,0.03 \pi)$, while the minimal value occurs at $\left(\sin \theta_{12}^{\ell}, \sin \theta_{13}^{\ell}, \sin \theta_{23}^{\ell}, \delta^{\ell}\right)=(0.9758,1.0000,0.8658,1.62 \pi)$, respectively.

\subsection{Constraining nonstandard neutrino interactions}

Let us study the phenomenological consequences of nonstandard neutrino interactions (NSIs) given in (3.9), (3.10), (3.11), and (3.12). For convenience we write down the effective operators responsible for the NSIs as [94-97]

$$
\mathcal{L}_{\mathrm{NSI}}=-2 \sqrt{2} G_{F} \epsilon_{\alpha \beta}^{f C}\left(\bar{\nu}_{\alpha} \gamma_{\mu} P_{L} \nu_{\beta}\right)\left(\bar{f} \gamma^{\mu} P_{C} f\right),
$$

where $\alpha, \beta$ denote the neutrino flavors $e, \mu$, and $\tau . P_{C}$ stands for the chiral projectors $P_{L}, P_{R}$. And, $f$ is the standard model first generation fermion $(e, u, d)$. The NSIs can affect neutrino oscillations in multiple ways, in the production, dectection and propagation of neutrinos. 
In this work, we consider the neutrino propagation in matter with the NSIs, assuming no effect of production and detection with the NSIs. The NSIs effect the neutrino propagation via coherent forward scattering in Earth matter. The Hamiltonian, which governs the propagation of neutrino flavor states in matter including the NSIs, is written as follows

$$
\hat{H}=\frac{1}{2 E}\left[\operatorname{UDiag}\left(m_{1}^{2}, m_{2}^{2}, m_{3}^{2}\right) U^{\dagger}+\operatorname{Diag}(A, 0,0)+A \epsilon^{m}\right],
$$

where $E$ is neutrino energy, $U$ is lepton mixing matrix, and $m_{i}$ are neutrino masses. Furthermore, $A=2 \sqrt{2} E G_{F} N_{e}$ is the effective matter potential that is driven by ordinary charged-current weak interaction with electron. $N_{e}$ is the electron density along the neutrino trajectory. The matrix $\epsilon^{m}$ has a form as

$$
\epsilon^{m}=\left(\begin{array}{ccc}
\epsilon_{e e} & \epsilon_{e \mu} & \epsilon_{e \tau} \\
\epsilon_{e \mu}^{*} & \epsilon_{\mu \mu} & \epsilon_{\mu \tau} \\
\epsilon_{e \tau}^{*} & \epsilon_{\mu \tau}^{*} & \epsilon_{\tau \tau}
\end{array}\right),
$$

where $\epsilon_{\alpha \beta}$ is called a matter NSI parameter and is defined as

$$
\epsilon_{\alpha \beta}=\sum_{f, C} \epsilon_{\alpha \beta}^{f C} \frac{N_{f}}{N_{e}},
$$

where $N_{f}$ is the number density of a fermion of type $f$. In the considering model, the form of $\epsilon_{\alpha \beta}^{f C}$ can be obtained as follows

$$
\begin{aligned}
\epsilon_{\alpha \beta}^{e L} & =\frac{\Gamma_{\alpha \beta}^{\nu Z^{\prime}} \Gamma_{e e}^{e Z^{\prime}}}{2 \sqrt{2} G_{F} m_{Z^{\prime}}^{2}}, \quad \epsilon_{\alpha \beta}^{e R}=\frac{\Gamma_{\alpha \beta}^{\nu Z^{\prime}}}{2 \sqrt{2} G_{F} m_{Z^{\prime}}^{2}}\left[\frac{g s_{W}^{2}}{c_{W} \sqrt{1+2 c_{2 W}}}\right], \\
\epsilon_{\alpha \beta}^{q L} & =-\frac{\Gamma_{\alpha \beta}^{\nu Z^{\prime}}}{2 \sqrt{2} G_{F} m_{Z^{\prime}}^{2}}\left[\frac{g\left(2+c_{2 W}\right)}{6 c_{W} \sqrt{1+2 c_{2 W}}}\right], \\
\epsilon_{\alpha \beta}^{q R} & =-\frac{\Gamma_{\alpha \beta}^{\nu Z^{\prime}}}{2 \sqrt{2} G_{F} m_{Z^{\prime}}^{2}}\left[\frac{g s_{W}^{2}}{3 c_{W} \sqrt{1+2 c_{2 W}}}\right] .
\end{aligned}
$$

The effective Hamiltonian in (4.48) governs the neutrino propagation in matter due to the NSIs, hence varying the neutrino oscillation probabilities in comparison to the normal case [98]. However, at present, there is no evidence for the NSI associated with the experimental data of neutrino oscillations. The latest constraints on the NSIs from the global analysis of oscillation data can be found in [99], which give the most stringent constraints on $\epsilon_{\alpha \beta}^{u}$ and $\epsilon_{\alpha \beta}^{d}$, namely $\epsilon_{\alpha \beta}^{u}$ bounded in the range $[-0.013,0.014]$ or $[-0.012,0.009]$ while $\epsilon_{\alpha \beta}^{d}$ bounded in the range $[-0.012,0.009]$ and $[-0.011,0.009]$, according to the cases of coherent data excluded or included, respectively.

From (4.51), we roughly estimate

$$
\left|\epsilon_{\alpha \beta}^{f C}\right| \sim \frac{1}{2 \sqrt{2} G_{F} m_{Z^{\prime}}^{2}} \simeq 3.0 \times 10^{-2}\left[\frac{1 \mathrm{TeV}}{m_{Z^{\prime}}}\right]^{2},
$$

which is at order $10^{-2}, 10^{-4}$, and $10^{-6}$ for $m_{Z^{\prime}}=1,10$, and $100 \mathrm{TeV}$, respectively. The predicted values lie within the experimental limits but do not give significant constraints on the model parameters. Comparing to the $\epsilon_{\alpha \beta}^{u}$ and $\epsilon_{\alpha \beta}^{d}$ bounds, it is hard to probe the nonstandard neutrino interactions in the model for $m_{Z^{\prime}}>3 \mathrm{TeV}$. 


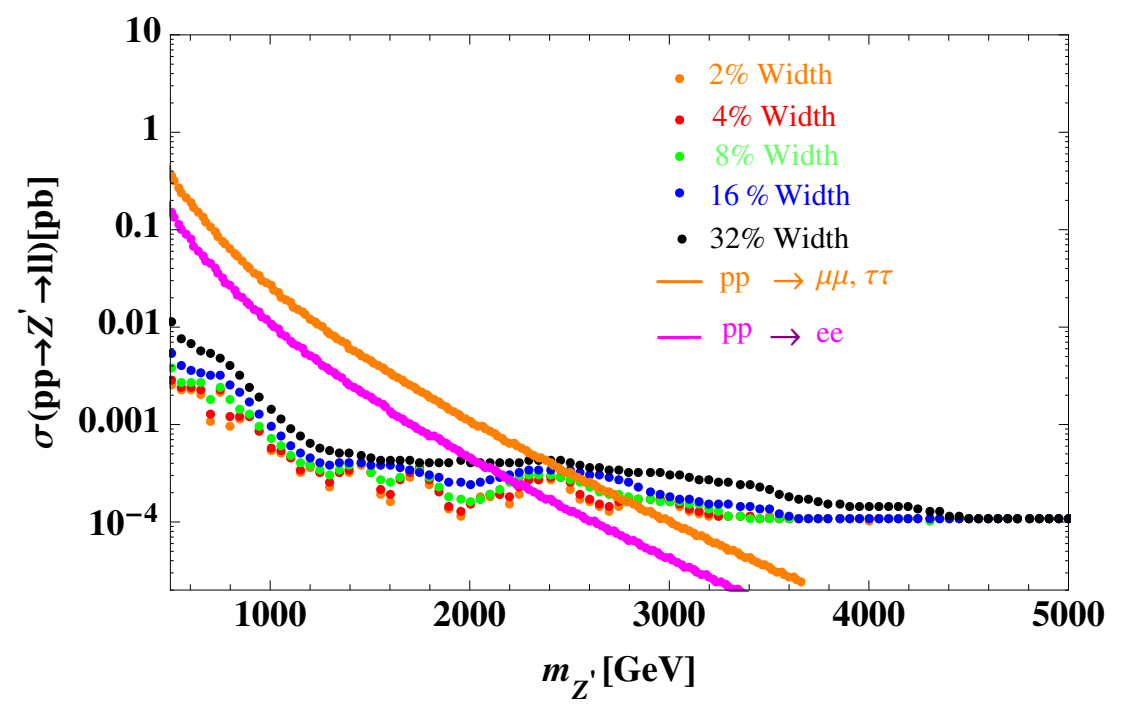

Figure 7. Dilepton production cross-section as a function of the new neutral gauge boson mass. The dotted lines are observed limits for different widths extracted at the resonance mass of dilepton, using $36.1 \mathrm{fb}^{-1}$ of $p p$ collision at $\sqrt{s}=13 \mathrm{TeV}$ by the ATLAS experiment [82].

\subsection{LHC dilepton and dijet searches}

Since the new neutral gauge boson $Z^{\prime}$ directly interacts with ordinary quarks $(q)$ and leptons $(l)$, the new physics processes $p p \rightarrow Z^{\prime} \rightarrow f \bar{f}$ for $f=q, l$ exist at the LHC dominantly contributed by $Z^{\prime}$.

The cross-section for producing a dilepton or diquark final state can be computed with the aid of the narrow width approximation [100],

$$
\sigma\left(p p \rightarrow Z^{\prime} \rightarrow f \bar{f}\right)=\frac{1}{3} \sum_{q} \frac{d L_{q \bar{q}}}{d m_{Z^{\prime}}^{2}} \hat{\sigma}\left(q \bar{q} \rightarrow Z^{\prime}\right) \operatorname{Br}\left(Z^{\prime} \rightarrow f \bar{f}\right)
$$

where the parton luminosities $d L_{q \bar{q}} / d m_{Z^{\prime}}^{2}$ at the $\mathrm{LHC} \sqrt{s}=13 \mathrm{TeV}$ can be found in [101]. The partonic cross-section and branching ratio $\operatorname{Br}\left(Z^{\prime} \rightarrow f \bar{f}\right)=\Gamma\left(Z^{\prime} \rightarrow f \bar{f}\right) / \Gamma_{Z^{\prime}}$ are

$$
\begin{aligned}
\hat{\sigma}\left(q \bar{q} \rightarrow Z^{\prime}\right) & =\frac{\pi g^{2}}{12 c_{W}^{2}}\left[\left(g_{V}^{Z^{\prime}}(q)\right)^{2}+\left(g_{A}^{Z^{\prime}}(q)\right)^{2}\right], \\
\Gamma\left(Z^{\prime} \rightarrow f \bar{f}\right) & =\frac{g^{2} m_{Z^{\prime}}}{48 \pi c_{W}^{2}} N_{C}\left[\left(g_{V}^{Z^{\prime}}(f)\right)^{2}+\left(g_{A}^{Z^{\prime}}(f)\right)^{2}\right], \\
\Gamma_{Z^{\prime}} & =\frac{g^{2} m_{Z^{\prime}}}{48 \pi c_{W}^{2}} \sum_{f} N_{C}\left[\left(g_{V}^{Z^{\prime}}(f)\right)^{2}+\left(g_{A}^{Z^{\prime}}(f)\right)^{2}\right],
\end{aligned}
$$

where $N_{C}$ is the color number of $f$ and assuming that $Z^{\prime}$ decays only to fermions. Indeed, it is easily verified that the other $Z^{\prime}$ decay channels such as to ordinary Higgs and gauge bosons give small contribution to the total $Z^{\prime}$ width.

In figure 7 , we show the cross-section for dilepton final states $l=e, \mu, \tau$. The experimental searches by the ATLAS [82] yield negative signals for new events of high mass, 
which transform to the lower limit for $Z^{\prime}$ mass, $m_{Z^{\prime}}>2.25$ and $2.8 \mathrm{TeV}$, according to ee and $\mu \mu(\tau \tau)$ channels, respectively. The last bound agrees with the highest invariant mass of dilepton hinted by the ATLAS. It is noteworthy that the $e e$ and $\mu \mu(\tau \tau)$ signal strengths are separated, which can be used to approve or rule out the flipped 3-3-1 model.

Furthermore, the dijet production cross-section for $q=u, d$ can be evaluated by comparing the $Z^{\prime} l \bar{l}$ and $Z^{\prime} q \bar{q}$ couplings. Indeed, since $\Gamma\left(Z^{\prime} \rightarrow u \bar{u}\right)=\Gamma\left(Z^{\prime} \rightarrow d \bar{d}\right) \simeq 2.3 \Gamma\left(Z^{\prime} \rightarrow\right.$ $e \bar{e}) \simeq \Gamma\left(Z^{\prime} \rightarrow \mu \bar{\mu}\right)=\Gamma\left(Z^{\prime} \rightarrow \tau \bar{\tau}\right)$, this leads to $\sigma\left(p p \rightarrow Z^{\prime} \rightarrow q \bar{q}\right) \sim \sigma\left(p p \rightarrow Z^{\prime} \rightarrow l \bar{l}\right)$. Because the current bound on dijet signals is less sensitive than the dilepton [83], the corresponding $Z^{\prime}$ mass limit is quite smaller than that obtained from the dilepton, which is not included. In sort, in the present model, the dijet sinals predicted are negligible, given that the dilepton bound applies for $Z^{\prime}$.

\subsection{Dark matter}

The model contains two kinds of dark matter candidates: (i) the fermion triplet $\xi$ which is unified with the standard model lepton doublet $\left(\nu_{1 L} e_{1 L}\right)$ in the $\mathrm{SU}(3)_{L}$ sextet and (ii) the scalar that is either $\rho_{3}$ or a combination (called $D$ ) of $\chi_{2}$ and $S_{23}$, whereas the remaining combination of $\chi_{2}$ and $S_{23}$ is the Goldstone boson of the $Y$ gauge boson. The candidate $D$ transforms as a standard model doublet, which interacts with $Z$. This gives rise to a large direct dark matter detection cross-section that is already ruled out [102]. The singlet candidate $\rho_{3}$ can fit the relic density and detection experiments, which has been studied extensively $[45,103]$. The fermion candidate $\xi$ is a new observation of this work. ${ }^{3}$

Note that at the tree-level, the components of $\xi$ triplet have degenerate masses, already obtained as $m_{\xi}$. However, the loop effects of gauge bosons can make the $\xi^{ \pm}$mass bigger than the $\xi^{0}$ mass by $m_{\xi^{ \pm}}-m_{\xi^{0}}=166 \mathrm{MeV}$ [104]. Therefore, $\xi^{0}$ can be regarded as a LWP responsible for dark matter.

In the early universe, the dark matter candidate $\xi^{0}$ can (co)annihilate into the standard model particles that set its abundance. Generalizing the result in [104], we obtain the annihilation cross-section,

$$
\begin{aligned}
\langle\sigma v\rangle & \simeq \frac{37 g^{4}}{96 \pi m_{\xi}^{2}} \\
& \simeq\left(\frac{\alpha}{150 \mathrm{GeV}}\right)^{2}\left(\frac{2.86 \mathrm{TeV}}{m_{\xi}}\right)^{2}
\end{aligned}
$$

where $(\alpha / 150 \mathrm{GeV})^{2} \simeq 1 \mathrm{pb}$. Comparing to the observation, we have $\Omega_{\xi} h^{2} \simeq 0.1 \mathrm{pb} /\langle\sigma v\rangle \simeq$ 0.11 [72], which implies $m_{\xi} \simeq 2.86 \mathrm{TeV}$.

The dark matter $\xi^{0}$ can scatter off nuclei causing observed effects in the direct detection experiments. At the tree-level, it does not interact with quarks confined in nucleons, since $T_{3}\left(\xi^{0}\right)=Y\left(\xi^{0}\right)=0$. The direct detection cross-section starts from the one-loop level, contributed by $W, h$ and $\xi^{ \pm}$, leading to $\sigma_{\mathrm{SI}} \simeq 1.2 \times 10^{-45} \mathrm{~cm}^{2}$ [105], in agreement with the experiment for the heavy dark matter mass $m_{\xi} \simeq 2.86 \mathrm{TeV}[106]$.

\footnotetext{
${ }^{3}$ In the minimal dark matter, such candidate was ruled out because a stability mechanism for dark matter was not appropriately taken [104].
} 
The above evaluation is valid when the new gauge $\left(Z^{\prime}\right)$ and Higgs $S_{33}$ portals as well as the 3-3-1 model new particles are heavier than the dark matter, so that the dark matter observables are governed by the standard model particles. Alternatively, since both $Z^{\prime}$ and $S_{33}$ couple to $\xi^{0}$, this dark matter can annihilate to the standard model Higgs, weak bosons and top quark as well as the appropriate new particles of the 3-3-1 model. In this case, the $Z^{\prime}$ and $S_{33}$ resonances set the dark matter density and direct detection crosssection $[43,45,103]$. That said, we have two viable regimes responsible for the dark matter mass $m_{\xi^{0}}=\frac{1}{2} m_{Z^{\prime}}$ and $m_{\xi^{0}}=\frac{1}{2} m_{S_{33}}$, provided that $m_{Z^{\prime}}$ and $m_{S_{33}}$ are separated. By contrast, these regimes are coincided. The $Z^{\prime}$ mass bound tells us that the dark matter mass is at $\mathrm{TeV}$ or higher scale. This mass is easily evaded the direct detection [106].

\section{Conclusion}

The discovery of the flipped 3-3-1 model has changed the current research of the 3-3-1 model. Indeed, the flavor nonuniversality that is now associated with leptons due to the anomaly cancellation provides the need for realizing the type I and II seesaw mechanism and the matter parity naturally. We have shown that the neutrino masses are produced, yielding the seesaw scales $\kappa$ at $\mathrm{eV}$ and $\mathcal{M}$ at $10^{14} \mathrm{GeV}$. Whereas, the dark matter candidate may be a fermion or scalar that has the mass in the $\mathrm{TeV}$ scale.

Because of the lepton generation nonuniversality, the charged lepton flavor violating processes and nonstandard neutrino interactions arise at the tree-level due to the exchange of $Z^{\prime}$ boson. We have made a systematic search for such processes and found that the 3-3-1 breaking scale is coincided with those obtained from the LHC dilepton and dark matter constraints, where the $Z^{\prime}$ portal governs the dark matter observables. This is an advantage over the ordinary 3-3-1 models since their FCNC constraints often do not coincide with the collision bounds $[45,53,66]$.

All the results arise from the gauge symmetry principles. Hence, the flipped 3-3-1 model is very predictive that deserves further studies.

\section{Acknowledgments}

P.V.D. and D.T.H. would like to thank Prof. Osamu Yasuda for the discussions on nonstandard interactions of neutrinos. This research is funded by Vietnam National Foundation for Science and Technology Development (NAFOSTED) under grant number 103.01-2017.05.

Open Access. This article is distributed under the terms of the Creative Commons Attribution License (CC-BY 4.0), which permits any use, distribution and reproduction in any medium, provided the original author(s) and source are credited.

\section{References}

[1] F. Pisano and V. Pleitez, An $\mathrm{SU}(3) \times \mathrm{U}(1)$ model for electroweak interactions, Phys. Rev. D 46 (1992) 410 [hep-ph/9206242] [INSPIRE]. 
[2] P.H. Frampton, Chiral dilepton model and the flavor question, Phys. Rev. Lett. 69 (1992) 2889 [INSPIRE].

[3] R. Foot, O.F. Hernandez, F. Pisano and V. Pleitez, Lepton masses in an $\mathrm{SU}(3)_{L} \times \mathrm{U}(1)_{N}$ gauge model, Phys. Rev. D 47 (1993) 4158 [hep-ph/9207264] [INSPIRE].

[4] M. Singer, J.W.F. Valle and J. Schechter, Canonical neutral current predictions from the weak electromagnetic gauge group $\mathrm{SU}(3) \times \mathrm{U}(1)$, Phys. Rev. D 22 (1980) 738 [inSPIRE].

[5] J.C. Montero, F. Pisano and V. Pleitez, Neutral currents and GIM mechanism in $\mathrm{SU}(3)_{L} \times \mathrm{U}(1)_{N}$ models for electroweak interactions, Phys. Rev. D 47 (1993) 2918 [hep-ph/9212271] [INSPIRE].

[6] R. Foot, H.N. Long and T.A. Tran, $\mathrm{SU}(3)_{L} \otimes \mathrm{U}(1)_{N}$ and $\mathrm{SU}(4)_{L} \otimes \mathrm{U}(1)_{N}$ gauge models with right-handed neutrinos, Phys. Rev. D 50 (1994) R34 [hep-ph/9402243] [INSPIRE].

[7] F. Pisano, A simple solution for the flavor question, Mod. Phys. Lett. A 11 (1996) 2639 [hep-ph/9609358] [INSPIRE].

[8] A. Doff and F. Pisano, Charge quantization in the largest leptoquark bilepton chiral electroweak scheme, Mod. Phys. Lett. A 14 (1999) 1133 [hep-ph/9812303] [INSPIRE].

[9] C.A. de Sousa Pires and O.P. Ravinez, Charge quantization in a chiral bilepton gauge model, Phys. Rev. D 58 (1998) 035008 [hep-ph/9803409] [INSPIRE].

[10] C.A. de Sousa Pires, Remark on the vector-like nature of the electromagnetism and the electric charge quantization, Phys. Rev. D 60 (1999) 075013 [hep-ph/9902406] [INSPIRE].

[11] P.V. Dong and H.N. Long, Electric charge quantization in $\mathrm{SU}(3)_{C} \times \mathrm{SU}(3)_{L} \times \mathrm{U}(1)_{X}$ models, Int. J. Mod. Phys. A 21 (2006) 6677 [hep-ph/0507155] [INSPIRE].

[12] P.B. Pal, The strong $C P$ question in $\mathrm{SU}(3)_{C} \times \mathrm{SU}(3)_{L} \times \mathrm{U}(1)_{N}$ models, Phys. Rev. D 52 (1995) 1659 [hep-ph/9411406] [INSPIRE].

[13] A.G. Dias, C.A. de S. Pires and P.S. Rodrigues da Silva, Discrete symmetries, invisible axion and lepton number symmetry in an economic 3-3-1 model, Phys. Rev. D 68 (2003) 115009 [hep-ph/0309058] [INSPIRE].

[14] A.G. Dias and V. Pleitez, Stabilizing the invisible axion in 3-3-1 models, Phys. Rev. D 69 (2004) 077702 [hep-ph/0308037] [INSPIRE].

[15] P.V. Dong, H.N. Long and H.T. Hung, Question of Peccei-Quinn symmetry and quark masses in the economical 3-3-1 model, Phys. Rev. D 86 (2012) 033002 [arXiv:1205.5648] [INSPIRE].

[16] M.B. Tully and G.C. Joshi, Generating neutrino mass in the 3-3-1 model, Phys. Rev. D 64 (2001) 011301 [hep-ph/0011172] [INSPIRE].

[17] A.G. Dias, C.A. de S. Pires and P.S. Rodrigues da Silva, Naturally light right-handed neutrinos in a 3-3-1 model, Phys. Lett. B 628 (2005) 85 [hep-ph/0508186] [INSPIRE].

[18] D. Chang and H.N. Long, Interesting radiative patterns of neutrino mass in an $\mathrm{SU}(3)_{C} \times \mathrm{SU}(3)_{L} \times \mathrm{U}(1)_{X}$ model with right-handed neutrinos, Phys. Rev. D 73 (2006) 053006 [hep-ph/0603098] [inSPIRE].

[19] P.V. Dong, H.N. Long and D.V. Soa, Neutrino masses in the economical 3-3-1 model, Phys. Rev. D 75 (2007) 073006 [hep-ph/0610381] [INSPIRE].

[20] P.V. Dong and H.N. Long, Neutrino masses and lepton flavor violation in the 3-3-1 model with right-handed neutrinos, Phys. Rev. D 77 (2008) 057302 [arXiv:0801.4196] [INSPIRE]. 
[21] P.V. Dong, L.T. Hue, H.N. Long and D.V. Soa, The 3-3-1 model with $A_{4}$ flavor symmetry, Phys. Rev. D 81 (2010) 053004 [arXiv:1001.4625] [InSPIRE].

[22] P.V. Dong, H.N. Long, D.V. Soa and V.V. Vien, The 3-3-1 model with $S_{4}$ flavor symmetry, Eur. Phys. J. C 71 (2011) 1544 [arXiv: 1009.2328] [INSPIRE].

[23] P.V. Dong, H.N. Long, C.H. Nam and V.V. Vien, The $S_{3}$ flavor symmetry in 3-3-1 models, Phys. Rev. D 85 (2012) 053001 [arXiv:1111.6360] [INSPIRE].

[24] S.M. Boucenna, S. Morisi and J.W.F. Valle, Radiative neutrino mass in 3-3-1 scheme, Phys. Rev. D 90 (2014) 013005 [arXiv:1405.2332] [InSPIRE].

[25] S.M. Boucenna, R.M. Fonseca, F. Gonzalez-Canales and J.W.F. Valle, Small neutrino masses and gauge coupling unification, Phys. Rev. D 91 (2015) 031702 [arXiv:1411.0566] [INSPIRE].

[26] S.M. Boucenna, J.W.F. Valle and A. Vicente, Predicting charged lepton flavor violation from 3-3-1 gauge symmetry, Phys. Rev. D 92 (2015) 053001 [arXiv:1502.07546] [INSPIRE].

[27] H. Okada, N. Okada and Y. Orikasa, Radiative seesaw mechanism in a minimal 3-3-1 model, Phys. Rev. D 93 (2016) 073006 [arXiv: 1504.01204] [InSPIRE].

[28] C.A. d.S. Pires, Neutrino mass mechanisms in 3-3-1 models: a short review, arXiv: 1412.1002 [INSPIRE].

[29] A.G. Dias, C.A. de S. Pires and P.S. Rodrigues da Silva, The left-right $\mathrm{SU}(3)_{L} \times \mathrm{SU}(3)_{R} \times \mathrm{U}(1)_{X}$ model with light, keV and heavy neutrinos, Phys. Rev. D 82 (2010) 035013 [arXiv:1003.3260] [INSPIRE].

[30] D.T. Huong and P.V. Dong, Left-right asymmetry and $750 \mathrm{GeV}$ diphoton excess, Phys. Rev. D 93 (2016) 095019 [arXiv: 1603.05146] [InSPIRE].

[31] M. Reig, J.W.F. Valle and C.A. Vaquera-Araujo, Unifying left-right symmetry and 3-3-1 electroweak theories, Phys. Lett. B 766 (2017) 35 [arXiv: 1611.02066] [InSPIRE].

[32] D. Fregolente and M.D. Tonasse, Selfinteracting dark matter from an $\mathrm{SU}(3)_{L} \times \mathrm{U}(1)_{N}$ electroweak model, Phys. Lett. B 555 (2003) 7 [hep-ph/0209119] [InSPIRE].

[33] H.N. Long and N.Q. Lan, Selfinteracting dark matter and Higgs bosons in the $\mathrm{SU}(3)_{C} \times \mathrm{SU}(3)_{L} \times \mathrm{U}(1)_{N}$ model with right-handed neutrinos, Europhys. Lett. 64 (2003) 571 [hep-ph/0309038] [inSPIRE].

[34] S. Filippi, W.A. Ponce and L.A. Sanchez, Dark matter from the scalar sector of 3-3-1 models without exotic electric charges, Europhys. Lett. 73 (2006) 142 [hep-ph/0509173] [INSPIRE].

[35] C.A. de S. Pires and P.S. Rodrigues da Silva, Scalar bilepton dark matter, JCAP 12 (2007) 012 [arXiv:0710.2104] [INSPIRE].

[36] J.K. Mizukoshi, C.A. de S. Pires, F.S. Queiroz and P.S. Rodrigues da Silva, WIMPs in a 3-3-1 model with heavy sterile neutrinos, Phys. Rev. D 83 (2011) 065024

[arXiv: 1010.4097] [INSPIRE].

[37] J.D. Ruiz-Alvarez, C.A. de S. Pires, F.S. Queiroz, D. Restrepo and P.S. Rodrigues da Silva, On the connection of gamma-rays, dark matter and Higgs searches at LHC, Phys. Rev. D 86 (2012) 075011 [arXiv:1206.5779] [inSPIRE].

[38] S. Profumo and F.S. Queiroz, Constraining the Z' mass in 3-3-1 models using direct dark matter detection, Eur. Phys. J. C 74 (2014) 2960 [arXiv:1307.7802] [InSPIRE]. 
[39] C. Kelso, C.A. de S. Pires, S. Profumo, F.S. Queiroz and P.S. Rodrigues da Silva, A 3-3-1 WIMPy dark radiation model, Eur. Phys. J. C 74 (2014) 2797 [arXiv:1308.6630] [INSPIRE].

[40] P.S. Rodrigues da Silva, A brief review on WIMPs in 3-3-1 electroweak gauge models, Phys. Int. 7 (2016) 15 [arXiv: 1412.8633] [INSPIRE].

[41] P.V. Dong, T.P. Nguyen and D.V. Soa, 3-3-1 model with inert scalar triplet, Phys. Rev. D 88 (2013) 095014 [arXiv:1308.4097] [INSPIRE].

[42] P.V. Dong, N.T.K. Ngan and D.V. Soa, Simple 3-3-1 model and implication for dark matter, Phys. Rev. D 90 (2014) 075019 [arXiv:1407.3839] [INSPIRE].

[43] P.V. Dong, C.S. Kim, D.V. Soa and N.T. Thuy, Investigation of dark matter in minimal 3-3-1 models, Phys. Rev. D 91 (2015) 115019 [arXiv:1501.04385] [INSPIRE].

[44] P.V. Dong, H.T. Hung and T.D. Tham, 3-3-1-1 model for dark matter, Phys. Rev. D 87 (2013) 115003 [arXiv:1305.0369] [INSPIRE].

[45] P.V. Dong, D.T. Huong, F.S. Queiroz and N.T. Thuy, Phenomenology of the 3-3-1-1 model, Phys. Rev. D 90 (2014) 075021 [arXiv:1405.2591] [InSPIRE].

[46] P.V. Dong, Unifying the electroweak and B-L interactions, Phys. Rev. D 92 (2015) 055026 [arXiv: 1505. 06469] [INSPIRE].

[47] D.T. Huong and P.V. Dong, Neutrino masses and superheavy dark matter in the 3-3-1-1 model, Eur. Phys. J. C 77 (2017) 204 [arXiv: 1605.01216] [INSPIRE].

[48] A. Alves, G. Arcadi, P.V. Dong, L. Duarte, F.S. Queiroz and J.W.F. Valle, Matter-parity as a residual gauge symmetry: probing a theory of cosmological dark matter, Phys. Lett. B 772 (2017) 825 [arXiv: 1612.04383] [INSPIRE].

[49] C.P. Ferreira, M.M. Guzzo and P.C. de Holanda, Cosmological bounds of sterile neutrinos in a $\mathrm{SU}(3)_{C} \otimes \mathrm{SU}(3)_{L} \otimes \mathrm{SU}(3)_{R} \otimes \mathrm{U}(1)_{N}$ model as dark matter candidates, Braz. J. Phys. 46 (2016) 453 [arXiv:1509.02977] [INSPIRE].

[50] P.V. Dong, D.T. Huong, F.S. Queiroz, J.W.F. Valle and C.A. Vaquera-Araujo, The dark side of flipped trinification, JHEP 04 (2018) 143 [arXiv:1710.06951] [INSPIRE].

[51] D.T. Huong, P.V. Dong, C.S. Kim and N.T. Thuy, Inflation and leptogenesis in the 3-3-1-1 model, Phys. Rev. D 91 (2015) 055023 [arXiv:1501.00543] [INSPIRE].

[52] P. Van Dong, D.T. Huong, D.A. Camargo, F.S. Queiroz and J.W.F. Valle, Asymmetric dark matter, inflation and leptogenesis from B-L symmetry breaking, Phys. Rev. D 99 (2019) 055040 [arXiv: 1805.08251] [INSPIRE].

[53] P.V. Dong, D.Q. Phong, D.V. Soa and N.C. Thao, The economical 3-3-1 model revisited, Eur. Phys. J. C 78 (2018) 653 [arXiv:1706.06152] [inSPIRE].

[54] D.J. Gross and R. Jackiw, Effect of anomalies on quasirenormalizable theories, Phys. Rev. D 6 (1972) 477 [INSPIRE].

[55] H. Georgi and S.L. Glashow, Gauge theories without anomalies, Phys. Rev. D 6 (1972) 429 [INSPIRE].

[56] J. Banks and H. Georgi, Comment on gauge theories without anomalies, Phys. Rev. D 14 (1976) 1159 [INSPIRE].

[57] S. Okubo, Gauge groups without triangular anomaly, Phys. Rev. D 16 (1977) 3528 [INSPIRE]. 
[58] R.M. Fonseca and M. Hirsch, A flipped 3-3-1 model, JHEP 08 (2016) 003 [arXiv: 1606.01109] [INSPIRE].

[59] D. Ng, The electroweak theory of $\mathrm{SU}(3) \times \mathrm{U}(1)$, Phys. Rev. D 49 (1994) 4805 [hep-ph/9212284] [INSPIRE].

[60] D. Gomez Dumm, F. Pisano and V. Pleitez, Flavor changing neutral currents in $\mathrm{SU}(3) \times \mathrm{U}(1)$ models, Mod. Phys. Lett. A 9 (1994) 1609 [hep-ph/9307265] [InSPIRE].

[61] H.N. Long and V.T. Van, Quark family discrimination and flavor changing neutral currents in the $\mathrm{SU}(3)_{C} \times \mathrm{SU}(3)_{L} \times \mathrm{U}(1)$ model with right-handed neutrinos, J. Phys. G 25 (1999) 2319 [hep-ph/9909302] [INSPIRE].

[62] A.J. Buras, F. De Fazio, J. Girrbach and M.V. Carlucci, The anatomy of quark flavour observables in 3-3-1 models in the flavour precision era, JHEP 02 (2013) 023 [arXiv:1211.1237] [INSPIRE].

[63] A.J. Buras, F. De Fazio and J. Girrbach, 3-3-1 models facing new $b \rightarrow s \mu^{+} \mu^{-}$data, JHEP 02 (2014) 112 [arXiv: 1311.6729] [INSPIRE].

[64] R. Gauld, F. Goertz and U. Haisch, An explicit $Z^{\prime}$-boson explanation of the $B \rightarrow K^{*} \mu^{+} \mu^{-}$ anomaly, JHEP 01 (2014) 069 [arXiv: 1310.1082] [INSPIRE].

[65] A.J. Buras and F. De Fazio, $\varepsilon^{\prime} / \varepsilon$ in 3-3-1 models, JHEP 03 (2016) 010 [arXiv: 1512.02869] [INSPIRE].

[66] P. Van Dong, N.T.K. Ngan, T.D. Tham, L.D. Thien and N.T. Thuy, Phenomenology of the simple 3-3-1 model with inert scalars, Phys. Rev. D 99 (2019) 095031 [arXiv:1512.09073] [INSPIRE].

[67] R. Delbourgo and A. Salam, The gravitational correction to PCAC, Phys. Lett. B 40 (1972) 381 [INSPIRE].

[68] L. Álvarez-Gaumé and E. Witten, Gravitational anomalies, Nucl. Phys. B 234 (1984) 269 [INSPIRE].

[69] A.G. Dias, R. Martinez and V. Pleitez, Concerning the Landau pole in 3-3-1 models, Eur. Phys. J. C 39 (2005) 101 [hep-ph/0407141] [INSPIRE].

[70] A.G. Dias, Evading the few TeV perturbative limit in 3-3-1 models, Phys. Rev. D 71 (2005) 015009 [hep-ph/0412163] [INSPIRE].

[71] P.V. Dong and H.N. Long, $\mathrm{U}(1)_{Q}$ invariance and $\mathrm{SU}(3)_{C} \times \mathrm{SU}(3)_{L} \times \mathrm{U}(1)_{X}$ models with $\beta$ arbitrary, Eur. Phys. J. C 42 (2005) 325 [hep-ph/0506022] [INSPIRE].

[72] Particle Data Group collaboration, Review of particle physics, Phys. Rev. D 98 (2018) 030001 [INSPIRE].

[73] P.V. Dong and D.T. Si, Kinetic mixing effect in the 3-3-1-1 model, Phys. Rev. D 93 (2016) 115003 [arXiv: 1510.06815] [INSPIRE].

[74] A.G. Dias, J.C. Montero and V. Pleitez, 3-3-1 models at electroweak scale, Phys. Lett. B 637 (2006) 85 [hep-ph/0511084] [INSPIRE].

[75] A.G. Dias, J.C. Montero and V. Pleitez, Closing the $\mathrm{SU}(3)_{L} \times \mathrm{U}(1)_{X}$ symmetry at electroweak scale, Phys. Rev. D 73 (2006) 113004 [hep-ph/0605051] [INSPIRE].

[76] T. Goto, Y. Okada and Y. Yamamoto, Tau and muon lepton flavor violations in the littlest Higgs model with T-parity, Phys. Rev. D 83 (2011) 053011 [arXiv:1012.4385] [INSPIRE]. 
[77] A. Ilakovac and A. Pilaftsis, Flavor violating charged lepton decays in seesaw-type models, Nucl. Phys. B 437 (1995) 491 [hep-ph/9403398] [INSPIRE].

[78] M. Blanke, A.J. Buras, B. Duling, A. Poschenrieder and C. Tarantino, Charged lepton flavour violation and $(g-2)_{\mu}$ in the littlest Higgs model with T-parity: a clear distinction from supersymmetry, JHEP 05 (2007) 013 [hep-ph/0702136] [INSPIRE].

[79] A.J. Buras, B. Duling, T. Feldmann, T. Heidsieck and C. Promberger, Lepton flavour violation in the presence of a fourth generation of quarks and leptons, JHEP 09 (2010) 104 [arXiv: 1006.5356] [INSPIRE].

[80] D.N. Dinh, A. Ibarra, E. Molinaro and S.T. Petcov, The $\mu-e$ conversion in nuclei, $\mu \rightarrow e \gamma$, $\mu \rightarrow 3 e$ decays and TeV scale see-saw scenarios of neutrino mass generation, JHEP 08 (2012) 125 [Erratum ibid. 09 (2013) 023] [arXiv: 1205.4671] [INSPIRE].

[81] D.N. Dinh and S.T. Petcov, Lepton flavor violating $\tau$ decays in TeV scale type I see-saw and Higgs triplet models, JHEP 09 (2013) 086 [arXiv:1308.4311] [INSPIRE].

[82] ATLAS collaboration, Search for new high-mass phenomena in the dilepton final state using $36 \mathrm{fb}^{-1}$ of proton-proton collision data at $\sqrt{\mathrm{s}}=13 \mathrm{TeV}$ with the ATLAS detector, JHEP 10 (2017) 182 [arXiv:1707.02424] [INSPIRE].

[83] ATLAS collaboration, Search for new phenomena in dijet events using $37 \mathrm{fb}^{-1}$ of $p p$ collision data collected at $\sqrt{s}=13 \mathrm{TeV}$ with the ATLAS detector, Phys. Rev. D 96 (2017) 052004 [arXiv: 1703.09127] [INSPIRE].

[84] A. Ilakovac, B.A. Kniehl and A. Pilaftsis, Semileptonic lepton number/flavor violating tau decays in Majorana neutrino models, Phys. Rev. D 52 (1995) 3993 [hep-ph/9503456] [INSPIRE].

[85] X.-G. He, J. Tandean and G. Valencia, Lepton-flavor-violating semileptonic $\tau$ decay and $K \rightarrow \pi \nu \bar{\nu}$, arXiv: 1904.04043 [INSPIRE].

[86] A. Cerri et al., Opportunities in flavour physics at the HL-LHC and HE-LHC, arXiv: 1812.07638 [INSPIRE].

[87] H.-B. Li and S.-H. Zhu, Mini-review of rare charmonium decays at BESIII, Chin. Phys. C 36 (2012) 932 [arXiv:1202.2955] [INSPIRE].

[88] Belle-II collaboration, The Belle II physics book, arXiv:1808.10567 [INSPIRE].

[89] L. Calibbi and G. Signorelli, Charged lepton flavour violation: an experimental and theoretical introduction, Riv. Nuovo Cim. 41 (2018) 71 [arXiv:1709.00294] [INSPIRE].

[90] SINDRUM II collaboration, Improved limit on the branching ratio of $\mu \rightarrow e$ conversion on lead, Phys. Rev. Lett. 76 (1996) 200 [INSPIRE].

[91] SINDRUM II collaboration, A search for muon to electron conversion in muonic gold, Eur. Phys. J. C 47 (2006) 337 [inSPIRE].

[92] COMET collaboration, A search for muon-to-electron conversion at J-PARC: the COMET experiment, PTEP 2013 (2013) 022C01 [INSPIRE].

[93] R. Kitano, M. Koike and Y. Okada, Detailed calculation of lepton flavor violating muon electron conversion rate for various nuclei, Phys. Rev. D 66 (2002) 096002 [Erratum ibid. D 76 (2007) 059902] [hep-ph/0203110] [INSPIRE].

[94] Y. Grossman, Nonstandard neutrino interactions and neutrino oscillation experiments, Phys. Lett. B 359 (1995) 141 [hep-ph/9507344] [INSPIRE]. 
[95] Z. Berezhiani and A. Rossi, Limits on the nonstandard interactions of neutrinos from $e^{+} e^{-}$ colliders, Phys. Lett. B 535 (2002) 207 [hep-ph/0111137] [InSPIRE].

[96] S. Davidson, C. Pena-Garay, N. Rius and A. Santamaria, Present and future bounds on nonstandard neutrino interactions, JHEP 03 (2003) 011 [hep-ph/0302093] [INSPIRE].

[97] T. Ohlsson, Status of non-standard neutrino interactions, Rept. Prog. Phys. 76 (2013) 044201 [arXiv: 1209.2710] [INSPIRE].

[98] D. Meloni, T. Ohlsson and H. Zhang, Exact and approximate formulas for neutrino mixing and oscillations with non-standard interactions, JHEP 04 (2009) 033 [arXiv:0901.1784] [INSPIRE].

[99] I. Esteban, M.C. Gonzalez-Garcia, M. Maltoni, I. Martinez-Soler and J. Salvado, Updated constraints on non-standard interactions from global analysis of oscillation data, JHEP 08 (2018) 180 [arXiv: 1805.04530] [INSPIRE].

[100] E. Accomando, A. Belyaev, L. Fedeli, S.F. King and C. Shepherd-Themistocleous, $Z^{\prime}$ physics with early LHC data, Phys. Rev. D 83 (2011) 075012 [arXiv: 1010.6058] [INSPIRE].

[101] A.D. Martin, W.J. Stirling, R.S. Thorne and G. Watt, Parton distributions for the LHC, Eur. Phys. J. C 63 (2009) 189 [arXiv:0901.0002] [InSPIRE].

[102] R. Barbieri, L.J. Hall and V.S. Rychkov, Improved naturalness with a heavy Higgs: an alternative road to LHC physics, Phys. Rev. D 74 (2006) 015007 [hep-ph/0603188] [INSPIRE].

[103] D.T. Huong, P.V. Dong, N.T. Duy, N.T. Nhuan and L.D. Thien, Investigation of dark matter in the 3-2-3-1 model, Phys. Rev. D 98 (2018) 055033 [arXiv: 1802.10402] [INSPIRE].

[104] M. Cirelli, N. Fornengo and A. Strumia, Minimal dark matter, Nucl. Phys. B 753 (2006) 178 [hep-ph/0512090] [INSPIRE].

[105] M. Cirelli and A. Strumia, Minimal dark matter: model and results, New J. Phys. 11 (2009) 105005 [arXiv: 0903.3381] [INSPIRE].

[106] XENON collaboration, First dark matter search results from the XENON1T experiment, Phys. Rev. Lett. 119 (2017) 181301 [arXiv:1705. 06655] [INSPIRE]. 